\title{
Adopting Exergy Analysis for use in Aerospace
}

\author{
David Hayes $^{1}$, Mudassir Lone ${ }^{2}$, James F Whidborne, ${ }^{3}$ \\ Cranfield University, Cranfield, MK43 OAL, United Kingdom \\ José Camberos 4 \\ U.S. Air Force Research Laboratory, Wright Patterson Air Force Base, Ohio 45433 \\ Etienne Coetzee ${ }^{5}$ \\ Airbus Operations, Bristol, BS99 7AR, United Kingdom
}

\begin{abstract}
Thermodynamic analysis methods, based on an exergy metric, have been developed to improve system efficiency of traditional heat driven systems such as ground based power plants and aircraft propulsion systems. However, in more recent years interest in the topic has broadened to include applying these second law methods to the field of aerodynamics and complete aerospace vehicles. Work to date is based on highly simplified structures, but such a method could be shown to have benefit to the highly conservative and risk averse commercial aerospace sector. This review justifies how thermodynamic exergy analysis has the potential to facilitate a breakthrough in the optimization of aerospace vehicles based on a system of energy systems, through studying the exergy-based multidisciplinary design of future flight vehicles.
\end{abstract}

Keywords: Exergy, Thermodynamics, Multi Disciplinary Optimisation

\section{Introduction}

Future commercial aircraft are likely to become more complex and highly integrated systems. As such, the powerplant, aerodynamics, stucture and aircraft sub-systems must be designed in a more holistic manner, where a common comparison metric is used for optimisation purposes. This paper proposes an extension to classic energy analysis, 5 known as exergy analysis which is conducted by the coupling of the first and second law of thermodynamics. A general introduction to the use of exergy within aerospace systems can be found in Doty [39, 40] as well as the published textbook by Camberos and Moorhouse [31], where the primary focus is that of military and hypersonic systems. This paper reviews additional published work, with insights being derived for how this method can be used specifically for the commercial aerospace industry, but with relevance to all aeronautics disciplines.

This review is written to advocate an alternative systems engineering approach to developing future commercial aircraft, where entropy and energy are united to provide additional design insight where traditional methods are restricted.

There is relatively little use of exergy analysis outside the thermodynamically dominant process of power generation in propulsion systems, where exergy is only applied at sub-system level and not as a unifying metric. This has left the method widely unknown within the aerospace community. A thought upheld by Nixon in saying [74]

\footnotetext{
${ }^{1}$ Research Student, Centre for Aeronautics, d.hayes@cranfield.ac.uk

${ }^{2}$ Lecturer, Centre for Aeronautics, m.m.lone@cranfield.ac.uk

${ }^{3}$ Reader, Centre for Aeronautics, j.f.whidborne@cranfield.ac.uk

${ }^{4}$ Aerospace Engineer, Aerospace Systems Directorate, jose.camberos@us .af .mil

${ }^{5}$ Future Projects Engineer, Airbus Operations, etienne.coetzee@airbus.com
} 


\begin{tabular}{|llll|}
\hline \multicolumn{2}{|l}{ Nomenclature } & & \\
$\eta_{a}$ & Aerodynamic efficiency $(-)$ & $g$ & Gravity $\left(\frac{m}{s^{2}}\right)$ \\
$\eta_{p}$ & Propulsion efficiency $(-)$ & $H$ & Enthalpy $(J)$ \\
$\eta_{s}$ & Structural efficiency $(-)$ & $L$ & Lift force $(N)$ \\
$\gamma$ & Ratio of specific heats $(-)$ & $M$ & Mach number $(-)$ \\
$\mathcal{V}$ & Volume $\left(m^{3}\right)$ & $m$ & Mass $(k g)$ \\
$W_{0}$ & Final aircraft weight $(k g)$ & $p$ & Pressure $(P a)$ \\
$W_{1}$ & Initial aircraft weight $(k g)$ & $Q$ & Heat $($ energy transfer $)(J)$ \\
$\psi$ & Flow exergy $(J)$ & $R$ & Range $(m)$ \\
$\rho$ & Air density $\left(\frac{k g}{m^{3}}\right)$ & $S$ & Entropy $\left(\frac{J}{K}\right)$ \\
$A$ & Area $\left(m^{2}\right)$ & $T$ & Temperature $(K)$ \\
$c_{T}$ & Thrust specific fuel consumption $\left(\frac{g}{s . k N}\right)$ & $t$ & Time $(s)$ \\
$D$ & Drag force $(N)$ & $U$ & Internal energy $(J)$ \\
$E$ & Energy $(J)$ & $V$ & Velocity $\left(\frac{m}{s}\right)$ \\
$E x$ & Exergy $(J)$ & $v_{\infty}$ & Free stream velocity $\left(\frac{m}{s}\right)$ \\
$F$ & Helmholtz free energy $(J)$ & $W$ & Work $($ energy transfer $)(J)$ \\
$G$ & Gibbs free energy $(J)$ & $w_{n}$ & Velocity vector $\left(\frac{m}{s}\right)$ \\
& & & \\
\hline
\end{tabular}

"Aeronautics is generally thought to be a mature discipline with little extra benefit to be obtained from further specialized research ... The only way that the science of aerodynamics can be made fertile again is to change the building blocks of knowledge"

Thus this review challenges the traditional force balance approaches of design, and propose an alternative global viewpoint of energy balance, by tracking the exergy flow and entropy generation in the system. This aims to lay the foundations for a novel physics based Multi-Disciplinary Optimisation (MDO) method to enable the development and realisation of next generation commercial aircraft and technologies, where systems are highly coupled.

To achieve this, the state of the art in the aerospace industry is discussed in Section 2, including a study of aircraft design and future trends, and assessing why an energy based approach may be applicable. Section 3 provides an overview of the mathematics behind exergy analysis, and provides additional references for those interested in a more in-depth understanding. Section 4 reviews how exergy analysis has been widely applied in the fields of propulsion and hypersonic systems, where conclusions are derived on the limitations of this analysis. Section 5 begins the debate on the wider application of exergy analysis to commercial aircraft and looks at how other authors have justified the use of exergy for aerospace applications. Sections 6 and 7 then review how exergy has been applied to date in aerodynamics and multi-disciplinary optimisation. The review concludes with some thoughts of how exergy could be applied in the future of aerospace systems and the impediments to adoption in Section 8 and 9.

\section{Design and Analysis of Commercial Aerospace Systems}

Traditional development splits the aircraft into various disciplines, examples being propulsion, aerodynamics and structures. Each of these aim to individually optimise discipline specific performance metrics, which are then nondimensionalised using contrasting methods to provide a comparison to other disciplines of an aircraft, as seen in the Breguet Range Equation. Jupp [60] and Lee [61] cite the use of the Breguet Range Equation [10], where the product 


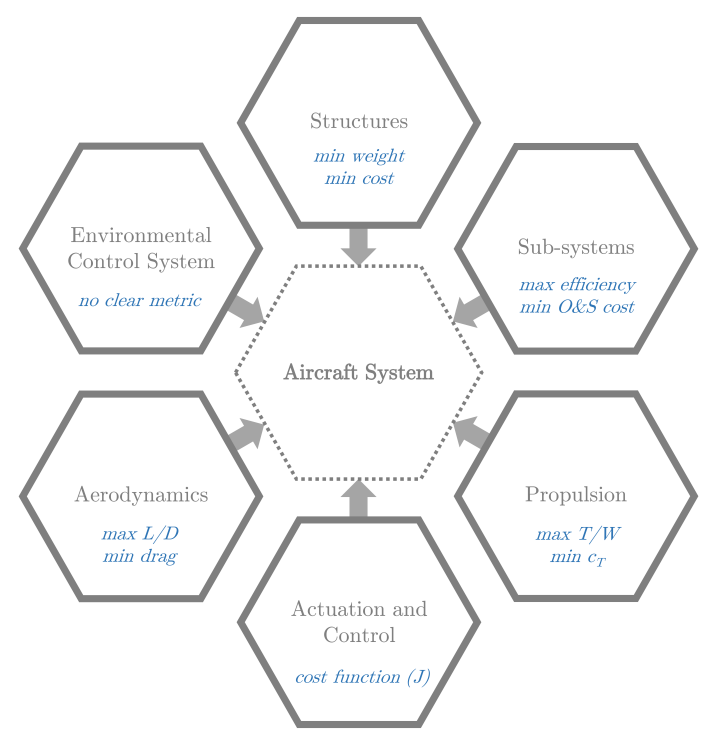

(a) Traditional discipline specific optimisation

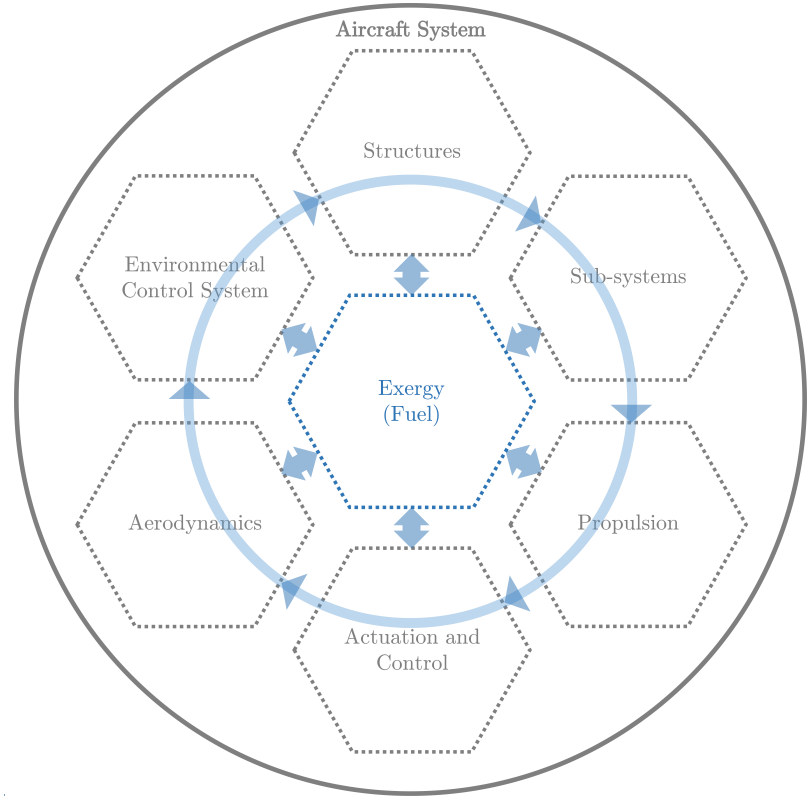

(b) Holistic system based optimisation

Figure 1: System Optimisation derived from Camberos [29]

of three non-dimensionalised relationships, the propulsion efficiency ${ }^{6}\left(\eta_{p}\right)$, the aerodynamic efficiency ${ }^{7}\left(\eta_{a}\right)$ and the structural efficiency ${ }^{8}\left(\eta_{s}\right)$, are optimised to maximise the aircraft range $(R)$ as

$$
R=\frac{v_{\infty}}{g} \underbrace{\frac{1}{c_{T}}}_{\eta_{p}} \underbrace{\frac{L}{D}}_{\eta_{a}} \underbrace{\ln \frac{\mathcal{W}_{1}}{\mathcal{W}_{0}}}_{\eta_{s}}
$$

In traditional design, and as a consequence of practicalities and difficulties in designing complex aerospace systems, there has been a historical segregation of component and sub-system design and analysis. This methodology has proved successful for conventional swept wing aircraft configurations, as the sub-system disciplines are only loosely integrated with one another. In addition, as a fallout of practicalities and work share in designing aerospace systems, there is a historical segregation of component and sub-system design and analysis. This means disciplines have individually developed performance, loss metrics and optimization at sub-system level and and are not clearly linked to the overall system performance metric or objective. So, when bought together to form the top level aircraft, as shown in Fig. 1a, it can be argued that even-though the system is optimised to a degree possible in terms of some system-level traditional performance metric as the interactions between these sub-systems has not been optimised the top level system will thus not be optimal.

From a systems engineer's perspective, their role at top level system design is to make trades between competing disciplines and sources of loss to arrive at a vehicle design with the least possible $\operatorname{cost}^{9}$. Thus the systems engineer has a need for a loss accounting method that enables systematic analysis of loss where system wide consequences of design trades can be evaluated. This premise led to the development of generalized models for vehicle thermodynamic loss management by Roth and Mavris [94], where differential loss models can be built of all aircraft sub-systems (Fig. 2 ), and the sources of work can be modelled against the vehicle losses under a unifying metric.

\footnotetext{
${ }^{6}$ Function of thrust specific fuel consumption $\left(c_{T}\right)$

${ }^{7}$ Function of aircraft Lift $(L)$ and total Drag $(D)$

${ }^{8}$ Function of initial aircraft weight $\left(\mathcal{W}_{1}\right)$ and final post fuel burn weight $\left(\mathcal{W}_{0}\right)$

${ }^{9}$ Typically in-flight fuel burn for a commercial aircraft
} 


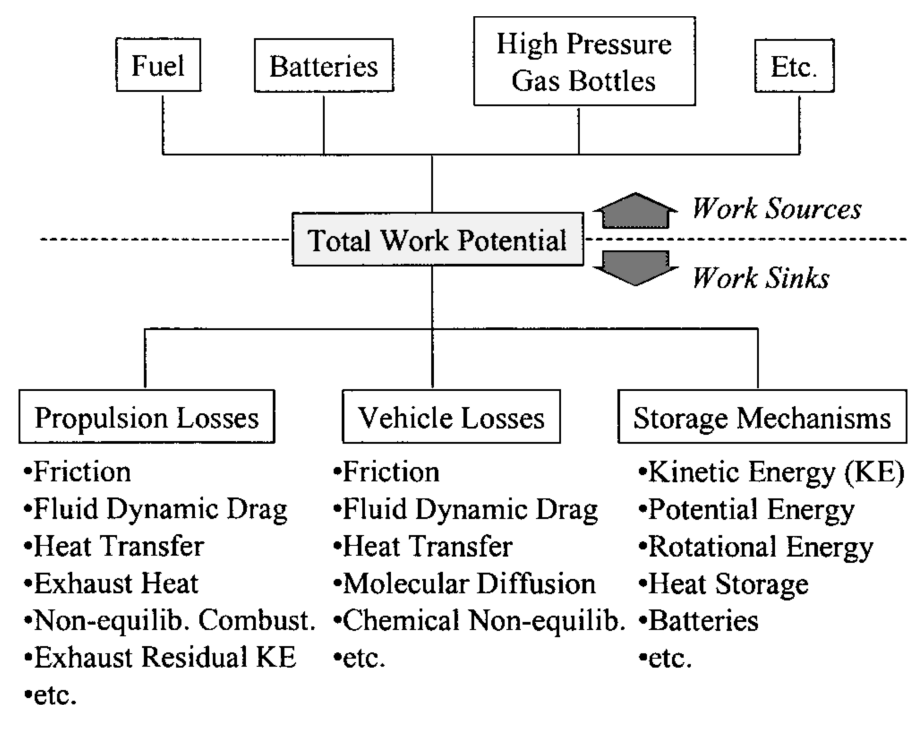

Figure 2: Typical contributers used in loss management model construction [94]

The question still remains though, of what unifying metric should be used in these loss models? The two universal properties proposed from thermodynamics are energy from the first law of thermodynamics and entropy from the second law of thermodynamics. Both energy and entropy are recognised as important in all natural processes including physics-based machines. Thus, using either of these metrics would provide the sought after integrated approach, as all aircraft systems operate using energy from a common fuel source and will generate entropy through their inefficiencies.

Any system design evolution works on the theory of allowing energy to transfer through a sub system more easily, by minimising the entropy production. Throughout the history of thermodynamics, the focus has been on heat engines and power generation technology with the aim to reducing the gap between actual operation and operation in the reversible cycle.

Current applications of thermodynamic analysis go well beyond power generation to near enough any system, with emphasis placed on identifying the mechanisms and system components that are responsible for thermodynamic losses (irreversibilities), through entropy generation. With this understanding, and the knowledge of the theoretical ideal operating conditions in a given environment, the concept of available work became the focus of academic study for thermodynamic optimisation, establishing the theoretical performance limit and maximum achievable efficiency of any system and process.

65 Thermodynamics is often misinterpreted in aeronautics as only being relevant to heat driven propulsion systems. However, given thermodynamics is the study of energy content and transfer, and all systems and processes use energy in some form, thermodynamic analysis provides a holistic approach to aircraft optimisation, suitable for all disciplines. Recall the four laws of thermodynamics [33]:

0. Zeroth Law If two bodies are in thermal equilibrium wih a third body, they are also in thermal equilibrium with each other

1. First Law Energy can be neither created of destroyed during a process; it can only change forms (principle of conservation of energy)

2. Second Law It is impossible for any device that operates on a cycle to receive heat from a single reservoir and produce a net amount of work (Kelvin-Planck statement)

3. Third Law The entropy of a pure crystalline substance at absolute zero temperature is zero, since there is no uncertainty about the state of the molecules at that instant

Thermodynamic exergy analysis, the method of this review, is the simultaneous application of the first and second laws. 


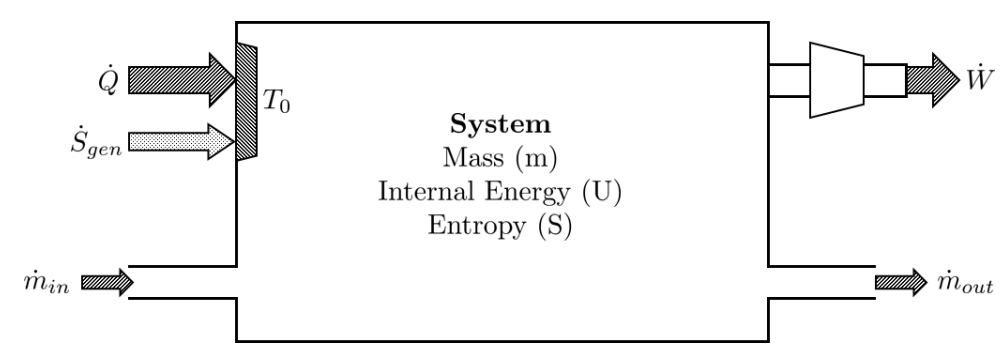

Figure 3: Sign convention for heat, work and mass transfer into a system [17]

\section{Thermodynamic Analysis for Commercial Aerospace Systems}

\subsection{First Law of Thermodynamics}

The first law of thermodynamics for a closed system allows energy to be transfered via work $(W)$ and heat $(Q)$, defined by the notation of Fig. 3, as

$$
\Delta E_{s y s}=\int Q d t-\int W d t
$$

which is derived by defining the Joule proportionality constant as the equivalence of work and heat, with the total sum of the energy remaining constant, such that energy is never created nor destroyed

$$
\dot{E}_{\text {sys }}=\dot{E}_{\text {in }}-\dot{E}_{\text {out }}
$$

However, for an energy study of a system such as an aircraft, the conversion of energy from one form to another is not only of interest, but also the conditions, limitations and direction of such a conversion. This is where the application of the second law of thermodynamics, the concept of entropy, can provide beneficial insight; as to whether the achieved final solution is near the optimal case or whether the solution is in fact feasible.

\subsection{Second Law of Thermodynamics}

Entropy is a useful state property in thermodynamics. If the process undergoes a thermodynamic transfer the entropy will either increase or remain the same. The latter case is known as a reversible process, where energy is transferred along a defined thermodynamic path cyclically such that the system returns to its initial state without any change, the so-called Carnot cycle for a frictionless heat engine. However a reversible process is an ideal case, as all real processes have irreversibilities, be it friction, expansion of gas, chemical reaction, diffusion of gases etc. Clausius' second law of thermodynamics, states that real processes are irreversible and proceed only in one particular sense. The directionality of a process can be defined in terms of the positive entropy generation, $S_{g e n}$ as a function of the systems pre, $S_{1}$, and post, $S_{2}$, entropy state, and that generated through heat transfer, $Q$, at a given temperature, $T$

$$
S_{\text {gen }}=S_{2}-S_{1}-\int \frac{\delta Q}{T} \geq 0
$$

Thus, even though energy can be transferred via work and heat, entropy generation is only associated with heat transfer. In effect the concept of entropy defines the limits of efficient energy transfer in the form of heat (heat engines). A systems total entropy, $S_{s y s}$, can be defined as

$$
\dot{S}_{\text {sys }}=\dot{S}_{\text {in }}-\dot{S}_{\text {out }}+\dot{S}_{\text {gen }}
$$




\subsection{Available Energy: The Synthesis of Energy and Entropy}

It can be postulated that when applied individually energy or entropy analysis are incomplete. Undertaking a first or second law analysis in isolation provides insight into the thermodynamic process behaviour, however neither sometimes considered an abstract concept as energy and entropy are thought to be separate state properties. However, the contrary is true, where a synthesised application allows the energy transfers of a system to be described with the first law, and the second law providing insight into the feasibility, directionality and the losses of useful energy with each of these energy transfers.

To clarify the understanding of a synthesised application take the coffee cup thought experiment. If a hot cup of coffee is placed in a cooler room environment, the first law of thermodynamics states that energy can be transferred via heat from the coffee cup to the environment, until the point at which the coffee and environment are in thermal equilibrium. However, the opposite also obeys the first law, where the environment transfers energy via heat to the coffee increasing the coffee temperature. When the second law is applied to this problem, and the definition that entropy transfer must always be positive, it is understood that the first transfer is the only feasible concept where energy is transferred from a hot to cold source.

The first mathematical synthesis of the first and second laws of thermodynamics was defined as available energy which was posed by Gibbs in 1873, a concept revised from a thermodynamic optimisation viewpoint by Gaggioli [49], [48]. Gibbs defined the term enthalpy

$$
H=U+p \mathcal{V}
$$

as a measure of the total energy of a system, the sum of the internal energy $(U)$ and the product of pressure $(p)$ and volume $(\mathcal{V})$. The Gibbs Free Energy $(G)$ is a theoretical value that defines the maximum work that can be obtained from a closed system undergoing a reversible, isothermal (constant temperature) and isobaric (constant pressure) process

$$
G=\underbrace{U+p \mathcal{V}}_{\text {First Law }}-\underbrace{T S}_{\text {Second Law }}
$$

A similar formulation is that of Helmholtz free energy $(F)$, which defines the maximum work that can be obtained from a closed system reversibly through a isothermal and isochoric (constant volume) process.

$$
F=U-T S
$$

\subsubsection{Exergy}

Exergy can be viewed as an extension of Gibbs and Helmholtz Free Energy where the available energy is not dependent on whether or not it is an isothermal, isobaric or isochoric process, however it is dependent on the unconstrained environment in which it resides. Ayres [15] highlights the similarity and difference of exergy to these two forms of free energy, as:

"The most general of all thermodynamic potentials of course is exergy, defined as the maximum amount of work that can be extracted from a system without any constraints on volume, pressure, temperature or composition." [15]

Understanding the two fundamental principles of thermodynamics, a combined definition from Sciubba [97], Bejan [19], Naterer [73] and Ayres [15] can propose a synthesised statement of the first and second laws.

"The maximum theoretical useful work obtained if a system is brought into thermodynamic equilibrium with the environment by means of processes in which the system interacts only with this environment. As such, exergy is a measure of the departure of the given state from the environmental state (distance from thermodynamic equilibrium), the larger the departure, the greater the potential for doing work. It is not a conserved quantity (like energy) but it is possible to construct an exergy balance for any energy or materials transformation process, accounting for inputs, process losses, useful products and wastes."

Thus analysis using the simultaneous application of the first and second law within a defined environment is defined as thermodynamic exergy analysis and its optimization component, entropy generation minimization (EGM). Bejan [19] comments that it is the premier method of thermodynamic analysis in engineering and it is now sweeping every aspect of engineering practice, including aerospace applications, emphasized by Camberos' statement that 
"Such capability [of exergy analysis] may allow the development of new and innovative concepts that do not just marginally improve performance but may enable the realization of entire new regimes of performance and operability, especially for high-speed aerospace vehicles" [30]

For additional information on the basic premise of exergy, the reader is directed to Edwards [42] who provides an uncomplicated thought experiment.

\section{Thermodynamic Analysis Coupling the First and Second Law}

This section outlines the general mathematics for use in exergy analysis, based on the previous definition.

\subsection{Environmental Definition}

The environment in which a system resides is more commonly known as a reference state, which is defined by thermodynamic variables of reference temperature $\left(T_{0}\right)$, reference pressure $\left(P_{0}\right)$ and reference chemical potential of species ' $k$ ' $\left(\mu_{k 0}\right)$. In practice an environment is defined by the baseline levels of any value, be it a temperature, pressure, velocity, chemical composition, altitude etc. However whilst all are required for exergy analysis the temperature is the most important as thermal energy transferred via heat is the least available form of energy, and the transfer associated with entropy generation.

\subsection{Physical (System) Exergy}

The exergy or available energy of an independent system residing in a given environment is defined by the systems physical exergy $\left(E x_{p h}\right)$. This can be easier understood by subdividing the total physical exergy into contributing parts, the terminology of which varies between authors, but for consistency with the rest of this body of work we shall view physical exergy as:

$$
E x_{p h}=E x_{U}+E x_{T}+E x_{V}+E x_{C}
$$

With the sub-exergies being defined as:

- Thermal exergy $\left(E x_{U}\right)$

- Internal exergy (closed system)

140

- Enthalpy exergy (open system)

- Kinetic Exergy $\left(E x_{T}\right)$

- Mechanical (objects in motion)

- Radiant (electromagnetic)

- Sound

- Potential Exergy $\left(E x_{V}\right)$

- Gravitational

- Stored mechanical (elastic)

- Nuclear

- Electrical

150

$$
\text { - Magnetic }
$$

- Chemical Exergy $\left(E x_{C}\right)$

Whilst chemical exergy is a form of potential exergy, it shall be discussed separately below due to the contrasting ideal conversion definition of potential exergy. 


\subsubsection{Thermodynamic exergy}

The thermodynamic exergy is defined as the work obtainable by taking the system through a process such as compression, expansion or heat exchange, to the temperature and pressure states of the environment [15]. For a closed system (non mass transfer) the thermodynamic exergy can be simplified to the internal exergy of the system

$$
E x_{U}=\left(U-U_{0}\right)+P_{0}\left(\mathcal{V}-\mathcal{V}_{0}\right)-T_{0}\left(S-S_{0}\right)
$$

where the maximum work that can be output is a function of the internal energy $(U)$, volume $\mathcal{V}$, entropy $(S)$ and the environment temperature $\left(T_{0}\right)$ and pressure $\left(P_{0}\right)$. However in an open system, the exergy of mass flow must be accounted for, as given in the enthalpy exergy

$$
E x_{U}=\underbrace{(U+P \mathcal{V})-\left(U_{0}+P_{0} \mathcal{V}_{0}\right)}_{\left(h-h_{0}\right)}-T_{0}\left(S-S_{0}\right)
$$

where a clear resemblance to Gibbs Free Energy (equation 7) can be seen, however in this case for a fully defined unconstrained environment.

\subsubsection{Kinetic exergy}

The kinetic exergy is defined as the work obtainable from movement, be it the motion of waves, electronics, atoms, molecules or substances.

$$
E x_{T}=\underbrace{\frac{1}{2} m\left(V-V_{0}\right)^{2}}_{\text {Mechanical }}
$$

\subsubsection{Potential exergy}

The potential exergy is defined as the work obtainable from system state (e.g. position, chemical composition, etc.), where the system has a disparity in some form to its environment which enables it to do or receive work.

$$
E x_{V}=\underbrace{m g\left(z-z_{0}\right)}_{\text {Gravitational }}+\underbrace{\frac{k\left(x-x_{0}\right)^{2}}{2}+\frac{\mathcal{V} E\left(\epsilon-\epsilon_{0}\right)^{2}}{2}}_{\text {Stored mechanical }} \underbrace{\frac{C\left(\Upsilon-\Upsilon_{0}\right)^{2}}{2}}_{\text {Electrical (capacitor) }}+\underbrace{\mathcal{M}\left(B-B_{0}\right)}_{\text {Magnetic }}
$$

In practice kinetic and potential exergy are both perfect forms of exergy, in that they can be completely converted to work, given a null reference state.

\subsubsection{Chemical exergy}

The chemical exergy is defined as the maximum amount of work obtainable by taking a system reversibly to the same chemical composition as the environment, with environmental temperature and pressure conditions. Camberos [31] formulates a mass derived chemical exergy (equal to the mole derived function) as given by

$$
E x_{C}=\sum_{i}^{n} y_{i}\left(\mu_{i_{1}}-\mu_{i_{0}}\right)
$$

where the exergy is a function of the chemical potential $\left(\mu_{k}\right)$ and mass ratio $\left(y_{i}\right)$ as opposed to alternative stoichiometric mole ratio $\left(v_{i, j} / v_{j}\right)$ relationships of Simpson [98].

Whilst this is a form of potential exergy, it is not a mechanical exergy, and thus not all of the energy can be converted to work. In addition to the exergy losses through heat generation (entropy production) found in reactions such as combustion, irreversibility is generated as environmental species are released to the environment at their environmental dead state chemical potential. 


\subsection{Exergy Transfer Equation}

Exergy (as with energy) can be transferred into and removed from a systems control volume though three means;

Where $\psi=\dot{E} x_{p h}=\dot{E} x_{U}+\dot{E} x_{T}+\dot{E} x_{V}+\dot{E} x_{C}$

\subsection{Entropy Generation and Exergy Destruction}

Independently from Gibbs' findings (equation 7), Gouy [54] and Stodola [102] defined the expression for useful energy in 1889 and 1898 respectively, as the difference between the enthalpy and the product of envionement temperature and entropy change. The principle of the increase of entropy is stated by Stodola as [102]:

"The sum of the entropies of all the bodies taking part in any [real] process whatever is at the end of the process greater than at its beginning"

The relationship stating entropy in terms of exergy destruction is important, as a value is generated in Joules, which allows losses due to entropy generation to be more easily understood. This is defined formally as the Guoy-Stodola theorem for exergy destruction $\left(E x_{d e s} \propto S_{g e n}\right)$

$$
E x_{d e s}=T_{0} S_{g e n}
$$

Defined by Stodola [102] as:

"For irreversible processes of any nature (also chemical), the useful work suffers a reduction equal to the product of the resulting increase of entropy in all the bodies taking part in the process and the temperature of the heat-abstracting reservoir, that is, the environment."

Bejan [17] proposes the name Entropy Generation Minimisation (EGM) for the process of multi-disciplinary optimistion using the exergy and exergy destruction metric. Unlike the first law range maximisation analysis in the Breguet equation (equation 1) exergy analysis and EGM are minimisation methods for exergy destruction and total exergy use. However it is worth noting a finite amount of friction (or some other entropy generation) is generally required for systems to operate. Consider ice skating in an ideal frictionless world, without friction with the ice there would be no way of propelling forward, thus no movement would happen.

\section{Performance Assessment}

The second law therefore provides additional insight to the first law analysis, in the following areas: 


\section{Applying Exergy Analysis to Aerospace Systems}

Exergy analysis results can be obtained by simply understanding how the components and their interactions are connected. Detailed information on the performance of a sub-system is not typically required, making it a useful tool from the preliminary design phase and through production. Exergy analysis provides " a consistent framework within which losses can be compared within machines, between machines of different types for the same job and with perfection in the form of the completely reversible machine" [34].

Thus it is achievable to formulate a set of vehicle design requirements stated in terms of the total energy use of the system, as an alternative view to that of discipline specific optimisation (see Fig. 1b). All systems over the flight mission can therefore be modelled in terms of the fuel energy used and exergy destruction losses. One of the convenient properties of exergy analysis is that exergy is easier interpreted than entropy, as exergy has the same units as energy (Joules), and as such in an economic analysis (also referred to as thermoeconomics in texts [18], [83] [84]) as a monetary value can be assigned to the loss of exergy in terms of energy cost, based on the cost of fuel per Joule for example. Then a simple comparison of every system can be made under the metric of exergy destruction in terms of fuel use.

All real world processes are irreversible. Auditing a design with the entropy approach will highlight where available energy is being used throughout the system, showing areas of unavoidable irreversibilities such as combustion losses as well as those irreversibilities with avoidable waste, as to direct the designers attention to those areas. The law approach is focused on identifying irreversibilties where entropy is produced with the aim to optimize the structure as to minimize this production. Some common examples of system generic irreversibilities are given as: Smith [99] 


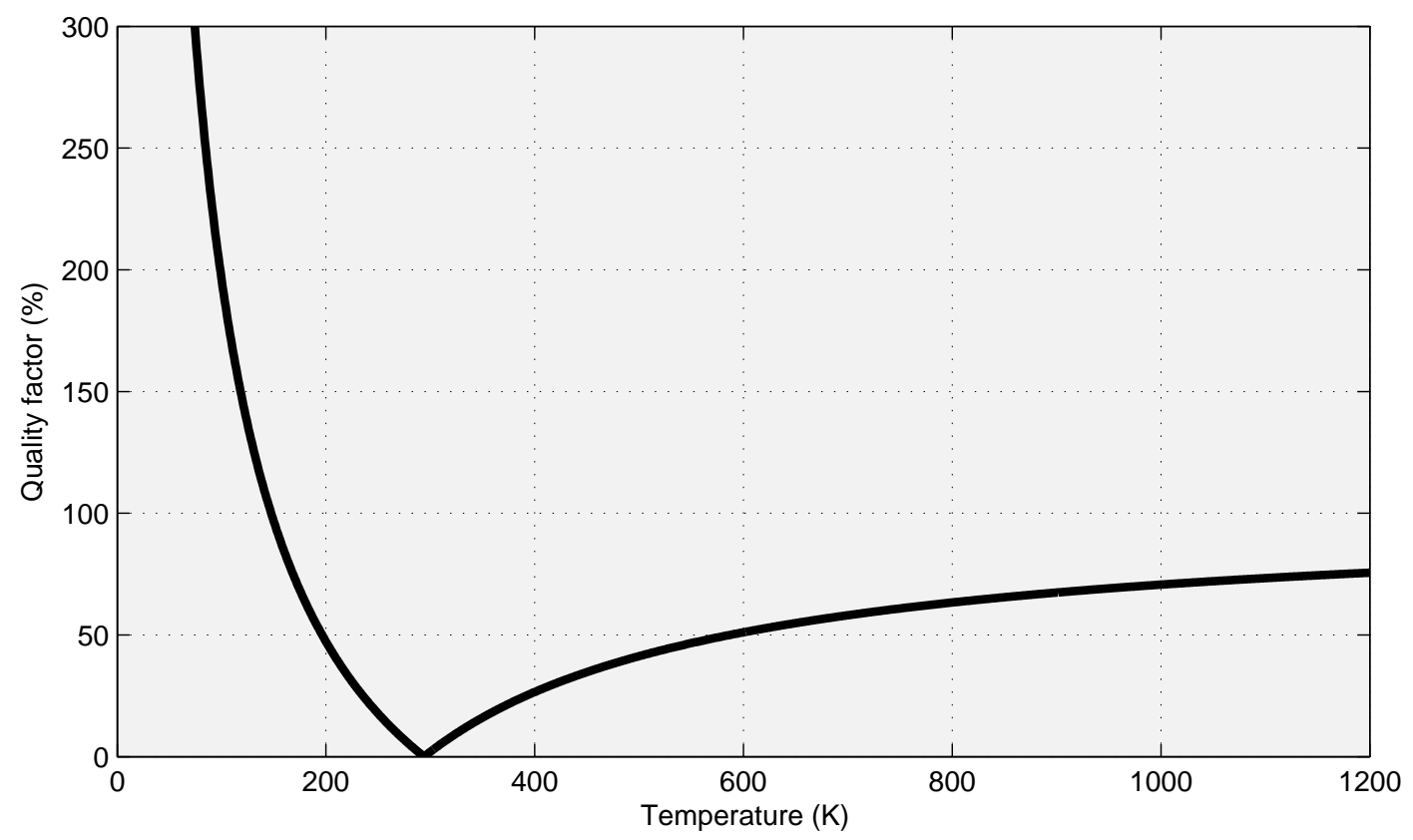

Figure 4: Energy Quality as a function of its temperature, modified from [15]

- Mixing objects or fluids

- Heat transfer (through a finite temperature difference)

- Friction as a result of relative motion of objects or fluids

- Chemical reactions

- Inelastic deformation of solids

- Electric resistance

- Drag (vortex and parasitic)

- Sudden compressions such as shock waves

The significance of exergy analysis research is shown by Giurgiutiu [52] who cites Moorhouse and his collaborators at the Air Force Research Laboratory (AFRL) studies into exergy-based multidisciplinary design as one of eight fundamental research projects for future flight structures. One of critical outputs from Moorhouse's work is the suggestion of changing the "analysis/design paradigm from energy-based to exergy-based (specifically, minimum exergy destruction). This shift in methodology is even more critical in exploratory research and development where previous experience may not be available to provide guidance" [39]. Hence, leading to the conclusion that the exergy-based conceptual framework enables the design of truly energy-efficient, integrated systems, subject to constraints.

Periannan $[79,80,81]$ applied exergy-based analysis and optimization methods to the synthesis/design and operation of aircraft systems to show the advantages of such a method over first law methods. This was done by comparing different objective functions to the same design; minimizing take-off gross weight, maximizing thrust efficiency, maximization of thermodynamic effectiveness, and minimizing exergy destruction. Periannan showed that:

"As long as the constraint space is the same, an energy-based objective produces the same optimum as that of the exergy-based objective provided that they are equivalent forms of the same thing, for example, fuel consumption" $[79]$

When this analysis was extended beyond propulsion and Environmental Control System (ECS) to include the aerodynamics (by definition not an energy system in the traditional sense), Periannan showed the equivalence between 
the energy and exergy objectives no longer holds. The need for a common currency points generally to the need for exergy as the basis for both analysis and optimization [80].

Doty [39] [41] [40] takes a similar comparison exercise, in this case a complex turbo-jet engine is simplified into interacting thermodynamic systems; the combustor as the energy source, the diffuser for energy transfer and the turbine for energy conversion. The paper aims to compare the same system process fromm an energy based first law method

$$
\dot{Q}-\dot{W}_{s}-\dot{W}_{\text {shear }}-W_{\text {other }}=\frac{\partial}{\partial t} \int_{C V} e \rho d V+\int_{C S}(e+p v) \rho \vec{V} d \vec{A}
$$

as previously given in equation 2 and an exergy based second law method

$$
\int_{C S}\left(1-\frac{T_{0}}{T_{k}}\right) \delta \dot{Q}-\left(\dot{W}-P_{0} \frac{d V_{C V}}{d t}\right)+\int_{C S} \psi \rho \vec{V}(d \vec{A})-\dot{E} x_{d e s}=\frac{d E x_{C V}}{d t}
$$

for the exergy transfer in an open system as in equation 15.

Three main conclusions are drawn from this comparison, which shows the advantages of the second law approach over the traditional first law methods [39]

- Second Law analysis provides physical limits on performance that the first law analysis does not

- First law energy analysis yields operating conditions that are not feasible, thus cannot exist. In the body of work, $40 \%$ of the results obtained from the first law analysis were not feasible.

- The exergy destruction focus provides a consistent accounting for all forms of losses regardless of point of origin

These examples have shown exergy analysis to be an excellent tool for optimising individual sub systems, however the true potential of exergy analysis in the integration of the different technical discipline, under a complete system of energy systems [30]. Optimization based on minimum exergy destruction, presents a Multi-Disciplinary Optimisation technique required for the analysis of aerospace vehicles in terms of the efficient use of on-board energy [30]. This style of analysis could be done at any stage of design on high or low fidelity models where the whole system is modelled and mapped over the entire mission profile and all locations of exergy destruction highlighted.

\subsection{Research Institutions focusing on Exergy}

From 2001 to 2009, the Air Force Office of Scientific Research funded research with the task; exergy based methods for aerospace vehicle design [52]. Camberos and Moorhouse have summarised much of this US Department of Defense funded research in Exergy Analysis and Design Optimization for Aerospace Vehicles and Systems [31]. The international research community applying exergy analysis to aerospace systems appears to be quite small, with Camberos and Moorhouse being involved (and leading) any US based research through various institutions including University of Dayton, Virginia Polytechnic Institute, Clemson University and Missouri University of Science and Technology, all of which have sizeable research groups working on exergy analysis. However there is plenty of interest in the topic outside of the US, with ONERA in France [12, 13, 14, 11], Anadolu University in Turkey [105, 43, 101], Canadian National Research Council [82],University of Sao Paulo in Brazil [35, 36, 51, 50], Cranfield University $[57,58]$ and Bath University $[23]$ in UK to name just a few.

\section{Exergy Analysis application to Propulsion systems}

Thermodynamic methods, such as exergy analysis, can provide a means for accounting for resources and wastes in a systematic uniform way. However application of these methods have been limited in application mainly to design optimisation of classical thermodynamics-based disciplines, thus have not seen much usage in other areas. Early exergy analyses were focused on extracting the maximum exergy from a hot gaseous stream which is discharged into the environment, as in an aircraft engine [18]. One of the reasons exergy research has focused heavily on thermodynamic dominant propulsion systems is the viewpoint that in comparison to exergy destroyed due to propulsion, all other forms of irreversibility are essentially negligible, thus focus for reduction through optimisation is on the engine [91].

From an exergy perspective, conventional turbofan engines convert chemical exergy into mechanical and electrical exergy tfor use by other aircraft systems. At the beginning of a flight the source of exergy for current passenger aircraft 

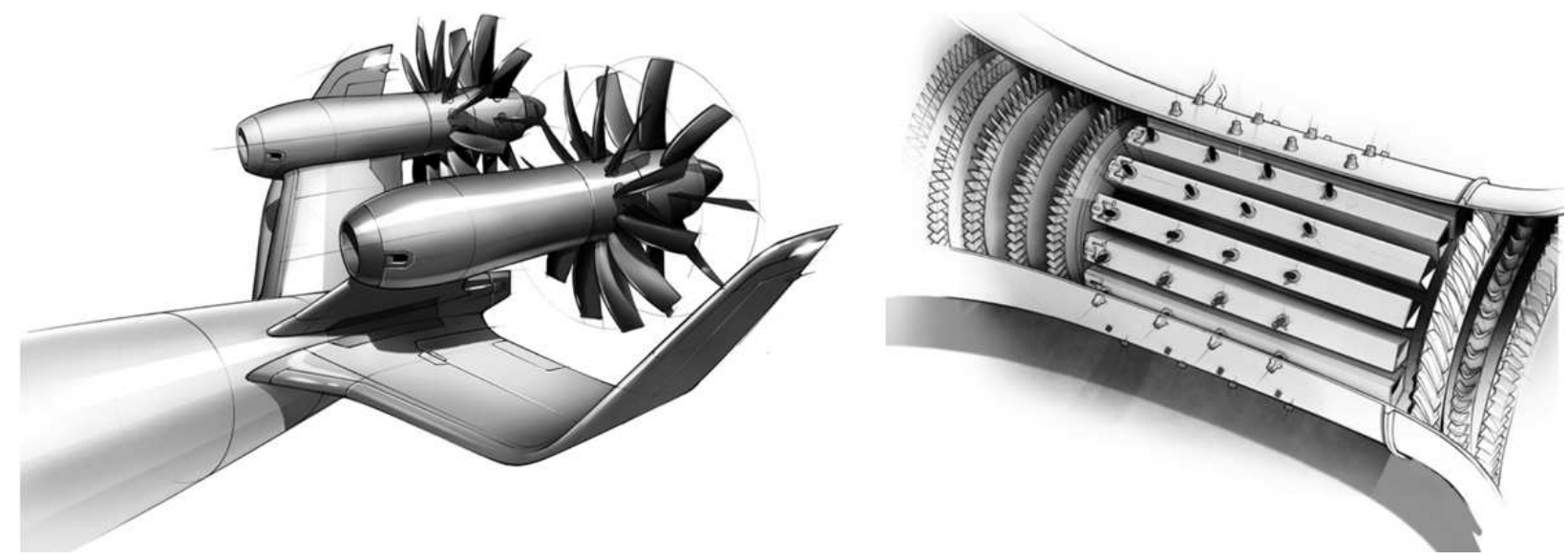

Figure 5: Chalmers University concepts, open rotor (left), pulse detonation core (right) [56]

is defined by the quantity of fuel and the chemical exergy contained within. Chemical exergy arises when there is a disequilibrium between the resource and environment leading to a chemical potential. This could be a potential created by a concentration gradient of species freely available in the environment, such as oxygen, carbon dioxide and methane, or exergy arises from a non-environmental species, fuels typically fall into this category. In both scenarios work can be extracted as the resource and environment are bought into chemical equilibrium.

Applying exergy analysis to aerospace systems is not a novel concept, with work dating back to the 1970s. The dominant field of interest in publications was applied to steady state propulsion systems, a clear extension to the previous applications of exergy to thermodynamic systems such as power stations. Examples of this work can be found in Sciubba [97], Glansdorff [53], Bauer [16], Maltry [65], Clarke \& Horlock [34], Lewis [63], Li \& Qiu [64], all of which undertook early optimisation analysis of aeronautic propulsive systems. This area is still under development today, with huge potential for improvement in efficiency of aircraft engines.

\section{"In time, the engines of nature acquire configurations that flow more easily, and this means that they evolve toward less entropy generation, and more production of motive power per unit of useful energy (exergy) used."[21]}

More recent approaches to applying exergy principles to the optimisation of propulsion systems, include Dincer [37], Clarke [34], Marley [68] and Ehyaei [43] in turbojet engines and Doty [41], Roth [93] [92] [95] and Riggins [85][88][86] in turbofan engines for commercial aircraft.

Noticing the lack of diversity in exergy analysis application beyond turbojet and turbofan, Grönstedt [56] included other potential future engines, using an assumed future 2050 optimised turbofan configuration as the baseline. Grönstedt performed an exergy analysis on an (futuristic) open rotor engine from Chalmers University, an intercooled recuperated engine and an engine working with a pulse detonation combustion core, which are the three alternative configurations he saw as the future of aircraft propulsion (see Fig. 5). Whilst Grönstedt showed the alternatives proposed provided a valid alternative to the turbofan configuration from an exergy perspective, what is more interesting are the conclusions on the use of the exergy metric, which were:

"A striking strength of the analysis is that it establishes a common currency for comparing losses originating from very different physical sources of irreversibility. This substantially reduces the complexity of analyzing and comparing losses in aero engines. In particular, the analysis sheds new light on how the intercooled recuperated engine establishes its performance benefits... As part of analyzing the computational results it has become evident that exergy analysis is also quite rewarding when a comparative analysis of different engine architectures is carried out.”[56]

An area exergy analysis may prove highly beneficial is in providing evidence for the integration of electric engines, an area of research gaining increasing focus for future aircraft. Schmitz [96] initially shows the shortcomings of traditional analysis methods, and then demonstrates how the unified figures of merit provided by exergy are useful in 
allowing for consistent comparisons between electric and conventional engines. Schmitz's work is concluded with a detailed comparison between a conventional turbofan, a parallel-hybrid turbofan, a novel integrated-hybrid turbofan concept, and an entirely electrical fan concept [96].

One should be mindful that individual sub-systems (such propulsion) optimised separately to the complete system are unlikely to be an optimised system as different sub-systems will have adverse effects on each other. So whilst use of exergy methods has been used for propulsion sub system optimisation, unless the integration of these to the complete system an optimised result will not be achieved. Justification for this top level systems approach can be seen from the analyses and optimisation of hypersonic vehicles (ramjet and scramjet) through exergy analysis by Brilliant [27], Markell [66] [67] and Tang [104]. What this work showed is that to fully realise the benefits of exergy analysis we must extend past just modelling the propulsion system to include the full system being mapped for its exergy uses, including application to the airframe and its losses through irreversibilities, providing a more holistic approach to the design process [96].

\section{Aerodynamic and Structural Optimisation using Exergy}

Concepts such as heat and work transfer are easily read across to aircraft systems such as propulsion systems or environmental control systems (ECS), however it is less clear how the second law analysis is used on purely aerodynamic systems. Exergy is transferred throughout the system including the airframe, but what needs to be identified are how the aerodynamics uses and converts exergy and the causes of entropy generation/exergy destruction. Taking a crude view of an airframe it has two primary purposes; (a) the structure to house the payload and (b) the aerodynamics to convert forward thrust to lift. It is common to take a force-based approach to the aircraft where the lift opposes the weight, and the drag opposed the thrust, giving the generalised coefficients

$$
\begin{aligned}
C_{L} & =\frac{L}{\frac{1}{2} \gamma P M^{2} A} \\
C_{D} & =\frac{D}{\frac{1}{2} \gamma P M^{2} A}
\end{aligned}
$$

The force balance approach, which results in lift and drag is just a theoretical concept to explain the basic aerodynamics of how a body flies, yet drag is simply a proxy for the second law entropy generation. Thus, an alternative theoretical concept viewpoint would be that of an energy approach where two key energies are calculated, that to keep the aircraft in the air, and that to propel the aircraft through the defined medium, in this case air. The airframe generated drag (contributions from vortex, parasitic and wave), accounts for a loss in useful energy. Whilst to minimise lift-induced drag, the vortex drag can be viewed as the entropy generation required to keep the aircraft in the air and thus cannot equal zero, whilst the parasitic and wave drag theoretically can be optimised to zero. Camberos derived the alternative exergy coefficient as a function of the entropy generation, comparable to that of the lift and drag coefficients, presented in Equation 21 is the corrected version from that of Camberos [29]

$$
C_{E x}=\frac{T_{0} \dot{S}_{g e n}}{\gamma P M^{2} v A}
$$

\subsection{The Oswatitsch expression for drag as an integral of entropy flow}

Early work in gas dynamics created the framework for deriving the entropy production through drag, notably from Oswatitsch. Oswatitsch [75] stated that drag was simply a generation of entropy, and thus the generation rate of such entropy would be the rate of exergy destruction.

"The power required to move a body immersed in a fluid with the constant velocity $u_{\infty}$ is equal to the temperature of the approach flow times the flow of entropy through an area which includes all entropy changes caused by the body" [75] 
In this case no useful work is done, $u_{\infty} W$ corresponds to the lost energy and the increase of the entropy flow represents the increase of entropy per unit time of the whole system [75], as

$$
\dot{E x}=v_{\infty} W=T_{\infty} \int\left(s-s_{\infty}\right) \rho w_{n} d f=T_{\infty} \int s \rho w_{n} d f
$$

Note that in the case of formation flight work is extracted from the drag of aircraft, so Oswatitsch above assumption may not be valid for multiple aircraft, unless the the aircraft group are considered a closed system and analyse the total entropy generated due to drag. Oswatitsch's statement is related to the Guoy-Stodola thermodynamic theorem (see equation 16) which states that the decrease of useful work of a thermal machine is equal to the entropy change of the system times the surrounding temperature.

Paulus [77] included the dead state velocity term as a method to account for the variable environment parameters in exergy mapping as

$$
\dot{E x}=F\left(\vec{V}-\vec{V}_{0}\right)=(T-D)\left(\vec{V}-\vec{V}_{0}\right)
$$

as well as continuing the work to develop an exergy definition for lift. An interesting application of Oswatitsch's integral of entropy flow was that of Greene [55], who showed through exergy methods that the ideal lift distribution for minimum induced drag is a parabolic shape and not the widely credited elliptical shape. Greene did this using the method as outlined by Oswatitsch [75], however his use of this method has been disputed and thus the result put into question [47] [89].

Entropy generation or exergy destruction due to aircraft aerodynamics are typically over shadowed by the exergy destruction within the propulsion system. This does not however mean there is no purpose to optimise aircraft aerodynamics, as it may be the case reducing exergy destruction due to drag is more cost effective than reducing total engine exergy destruction. Exergy analysis also proves to be a useful tool for wing optimisation when the aerodynamics are considered in isolation from the rest of the aircraft.

Significant resources have been put into optimising aerofoil shape and wing lift distribution to maximise lift to drag ratio, with these activities continuing to date for all new aircraft. Exergy analysis can be of benefit in improving the thermodynamic performance of the system by highlighting the mechanisms generating entropy and allowing the designer to pinpoint areas for improvement, or help dump unwanted energy from the system in landing or gust events.

Given the wide adoption of Computational Fluid Dynamics (CFD) in the design of aerodynamic systems, integration of exergy analysis into CFD solvers or as a post processor is an important step to make exergy analysis fully versatile. Where a Reynolds Averaging Naviour Stokes (RANS) approach is used to model the turbulent flow, Adeyinka [1] has included the modelled entropy production into the Navier-Stokes equations. The constitutive form of entropy generation, which is mathematically equivalent to the transport equation for entropy, is given by Alabi $[5,6,7,9,8]$ as

$$
\dot{S}_{g e n}=\frac{1}{T} \tau_{i j} \frac{\partial u_{i}}{\partial x_{j}}-\frac{q_{k}}{T^{2}} \frac{\partial T}{\partial x_{k}}
$$

which is used to calculate the entropy in the flow over the airframe sub-system aerodynamics (AFS-A) of a Boeing 747-200, cruising at Mach 0.855. Modelling both inviscid and viscous flow, Alabi concluded that for the inviscid solver minimal artificial entropy is produced, and that the majority of entropy generation is due to turbulence, as the viscous dissipation term in the entropy equation dominates compared to the heat transfer term. Examples of the output entropy generation are given in Fig. 6. Alabi validated this work using Prandtl-Glauret airfoil theory for a lumped parameter model $[5,6]$.

Focusing on the Blended Wing Body NASA N3-X configuration, Arntz [11, 13, 12], showed the same conclusion as Alabi in that viscous dissipation dominates entropy generation in drag. Arntz's work also investigated the exergy analysis of a blended wing boundary layer ingestion (BLI) system, and identified components of recoverable exergy in the wake/jet of the aircraft that could be recovered using BLI methods. This goes against the Oswatisch theory where all drag is lost energy and thus destroyed exergy.

Using a FORTRAN Reynolds-averaged Navier Stokes flow solution, Arntz [14] computed the entropy generation around the NASA Common Research Model (CRM) configuration travelling at transonic speed, thus as well as the parasitic and vortex drag components of entropy generation, Arntz was able to show the entropy generated in shock waves. Replicating and extending the work of Arntz at ONERA [14] McGuire [69] used the NASA Common Research Model (CRM) to calculate the entropy and exergy destruction in the induced, parasitic and wave drag, calculating the 

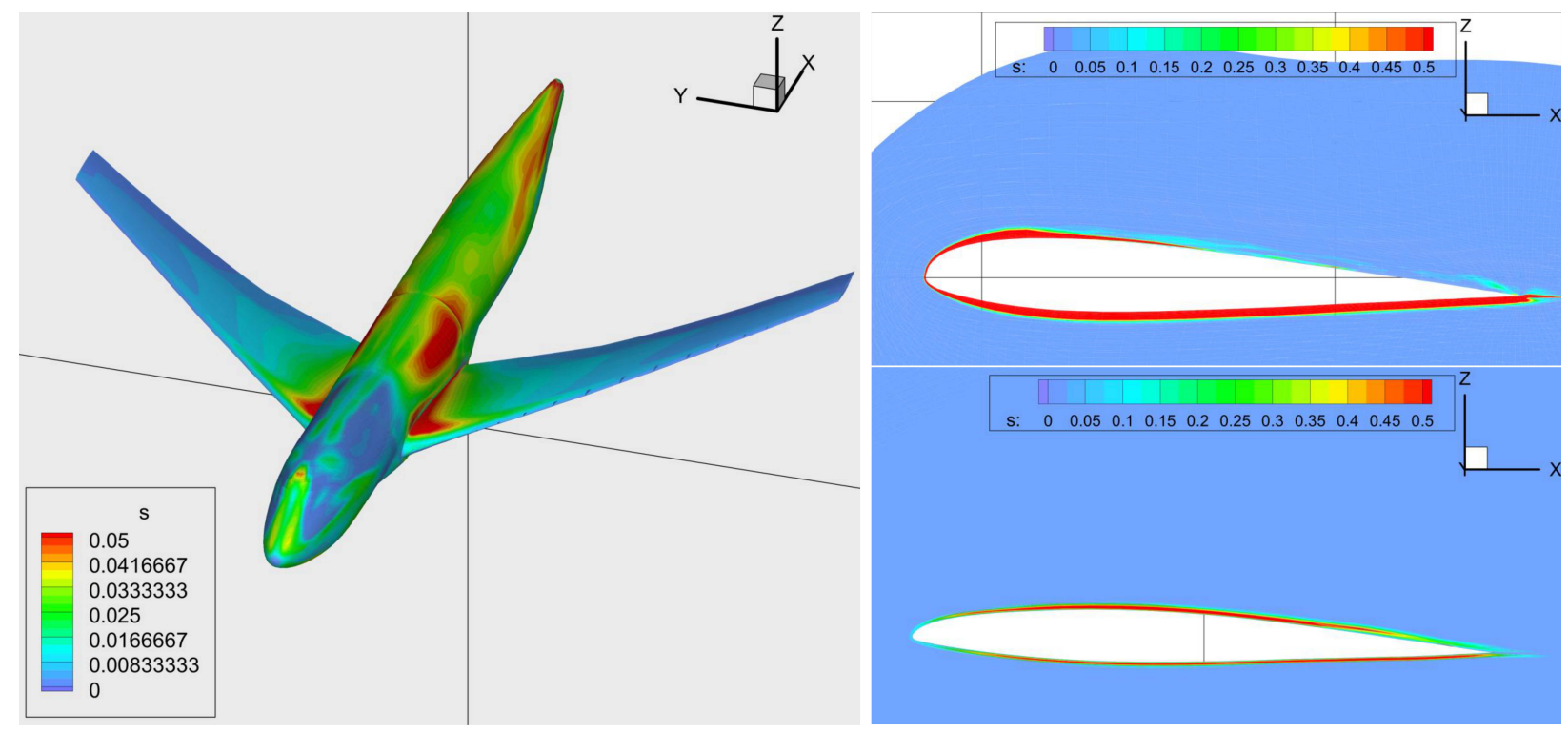

Figure 6: Entropy generation around the B747-200 aircraft and wing locations 14\% (upper) and 28\% (lower) [6]

power loss (exergy destruction rate) for the CRM cruising at transonic speeds. Some examples of the results from McGuire are given in Fig. 7.

Memon [70] provides a more detailed study for the exergy destruction in vortex drag, through experiments in a water tunnel at Institute of Aerospace Systems, focusing on the exergy distribution in the vortex for a variable angle of attack. When considering aerodynamics in energy terms, the point of minimum exergy state would be assumed to correspond to the maximum lift-to-drag ratio angle of attack. What Memon showed in that this is not the case, and that it is related to where the wing-tip vortex changes from a wakelike to jetlike vortex. Thus Memon concludes that "the exergy method holds promise as a metric for the improvement of aircraft performance through the reduction of lift- induced [drag]" [70].

Hayes [58] compared the use of implicit (the Breguet Range Equation) and second law explicit energy (Exergy Analysis) analysis methods for the incorporation of span extended technologies into future aircraft configurations. Hayes showed exergy analysis leads to a methodology that can support the design of the complete vehicle as a system of systems in a common mathematical framework. A critical part of this is the development of a decomposition strategy where all the subsystem components can be optimized to a system-level common metric. Critically, it was shown that both the Breguet and the Exergy method provide the same output from the benefit analysis when comparing different in-flight morphing mechanisms under each methodology. This can be seen in Fig. 8 where the analysis shows for an aspect ratio of 12.6 assuming the mass increase is less than $17 \%$ from the baseline 9.4 aspect ratio, an improvement of $11.5 \%$ can be made in the range which is equivalent to a reduction of up to $5 \%$ of the total source exergy (fuel) use. In addition, the exergy method provides a more detailed analysis method which allows energy losses to be compared to any of the aircraft's subsystems.

\section{Multi-Disciplinary Optimisation using the Exergy Metric}

Multi-disciplinary integrated design is where we consider the system as the complete collection of sub-systems interacting with one another and the optimisation of this system. As previously commented, this removes the issues with designing sub-systems in isolation where a sub-optimal design is usually the outcome.

Conventional Multi-Disciplinary Optimisation uses normalized coefficients to local variable dimensions, as seen in the Breguet equation (equation 1) with the aerodynamic, propulsion and structure non-dimensionalised factors. However, such a method cannot account for essential differences between the aerodynamic, propulsion and structural, and the magnitude of sensitivities can mislead the direction of optimization. Riggins [31] summarised this thought as 


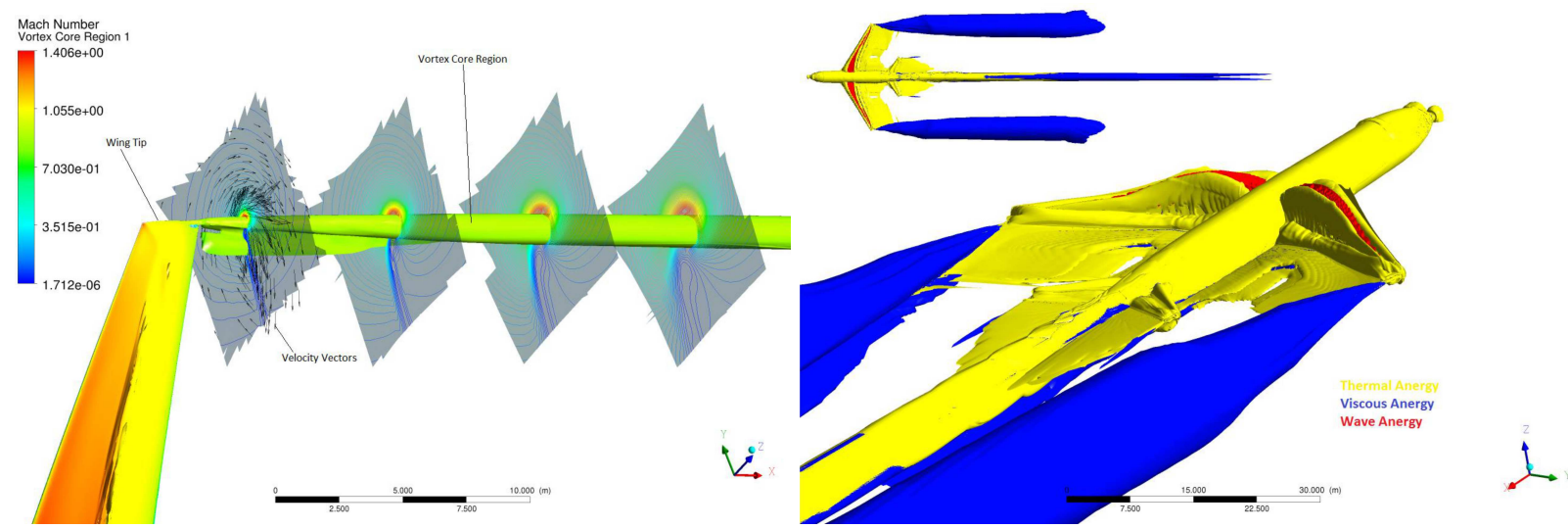

(a)

(b)

Figure 7: Entropy generation due to vortex, parasitic and wave drag [69]

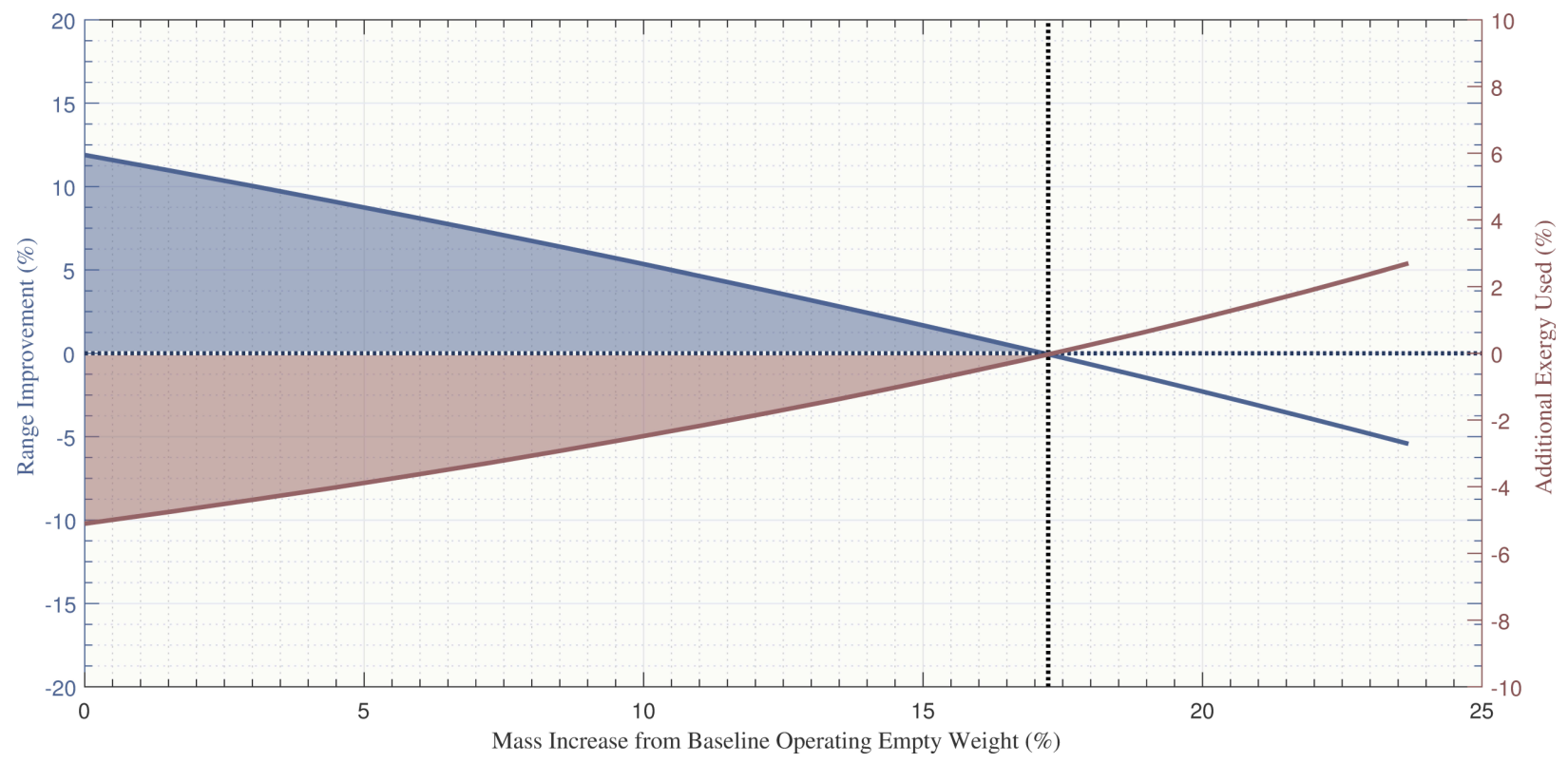

Figure 8: Comparison of the exergy and Breguet results for folding wing tips of an aspect ratio 12.6 aircraft [58] 
"In current Multi-Disciplinary Optimisation and Analysis efforts, the various components and subsystems are still generally evaluated or analysed in terms of their traditional and unique loss and performance metrics... the characteristic, property, or quantity being used as the metric for loss minimization at all levels of system, sub-system, and component design/evaluation should be the same as or at least explicitly related to the system-level performance objective itself"

There is no end point with the evolution of aerospace vehicles where one would say we have a perfect design, but what exergy offers is a tool to be used to highlight areas of designs that waste useful energy and thus could be improved to as near optimisation as feasible, summarised by Bejan as:

"Thermodyanmic optimisation (or entropy generation minimisation) brings the design as closely as permissible to the theoretical limit” [20]

Camberos defines some of the key advantages of the exergy true common currency objective function for MDO 415 as:

- Sensitivities are normalized according to global dimensions

- The magnitude of these sensitivities will be a better indication as to best direction for system optimization

- Opens viable (excluding physically infeasible) possibilities for revolutionary design

- Provide a clear picture of total system integration

By devising ways to avoid the destruction of exergy, better use can be made of fuels. By accounting for all the exergy streams of the system it is possible to determine the second law (exergetic) efficiency. By performing exergy accounting at higher and higher fidelities, a map can be drawn of how the destruction of exergy is distributed over the engineering system of interest. In this way the components and mechanisms (processes) that destroy exergy the most can be identified. It is then by repeating the exergy analysis on the improved system that one can evaluate the thermodynamic improvements made due to the second law implementation.

\subsection{Visualising Exergy Use}

Due to the complex nature of aircraft systems, many authors have commented on the difficulty in visualising exergy flow through an entire system. Two method have been proposed, the use of exergy flow diagrams from the work of Paulus [76] [77] and Grassman diagrams by De Oliveira [36] and Berg [22].

Fig. 9 shows an example exergy flow diagram from the work of Paulus. The diagram represents the interactions between different sub systems through which exergy can flow. One concern with this style of diagram is that with a more complex system such as a commercial aircraft (see Fig. 10) the diagram will quickly become cluttered with multiple interactions, making it difficult to decipher.

It is also easy to visualise the exergy flow through a system, using a similer graph to that of a Sankey Diagram, known as a Grassmann diagram, where the exergy source (typically fuel) is mapped throughout the flight mission to highlight areas of exergy destruction. The same exergy flow as in Fig. 10 can be shown for an A340 as an entropy flow diagram in Fig. 11 (equivalent to Fig. 9) and a Grassmann diagram in Fig. 12, where the horizontal arrow represents the flow of exergy, and the vertical arrows represent entropy production or exergy destroyed through various energy conversion processes, such as combustion, the Environmental Control System (ECS) and in the generation of drag [39].

\subsection{Full Vehicle Exergy Analysis}

Complete aircraft optimisation is the process of performing an exergy analysis over the complete flight profile and assessing the exergy flow and use throughout the system, to the point of total exergy destruction. A widely published and referenced body of work detailing an application complete aircraft exergy analysis is the morphing wing optimisation of a future advanced air to air fighter (AAF) [106, 100, 99, 79, 80, 81, 28, 26, 82, 83, 84, 66, 72, 71, 78]. This is an example of a widely applied application of exergy analysis where it is coupled with large-scale optimisation of a system, the principles of which are the same as discussed in previous sections.

The initial study into the AAF by Von Spakovsky [106] simplified the AAF into two sub-systems, the propulsion and airframe. The aim of the study, based on the DARPA morphing aircraft structures programme, was to perform 


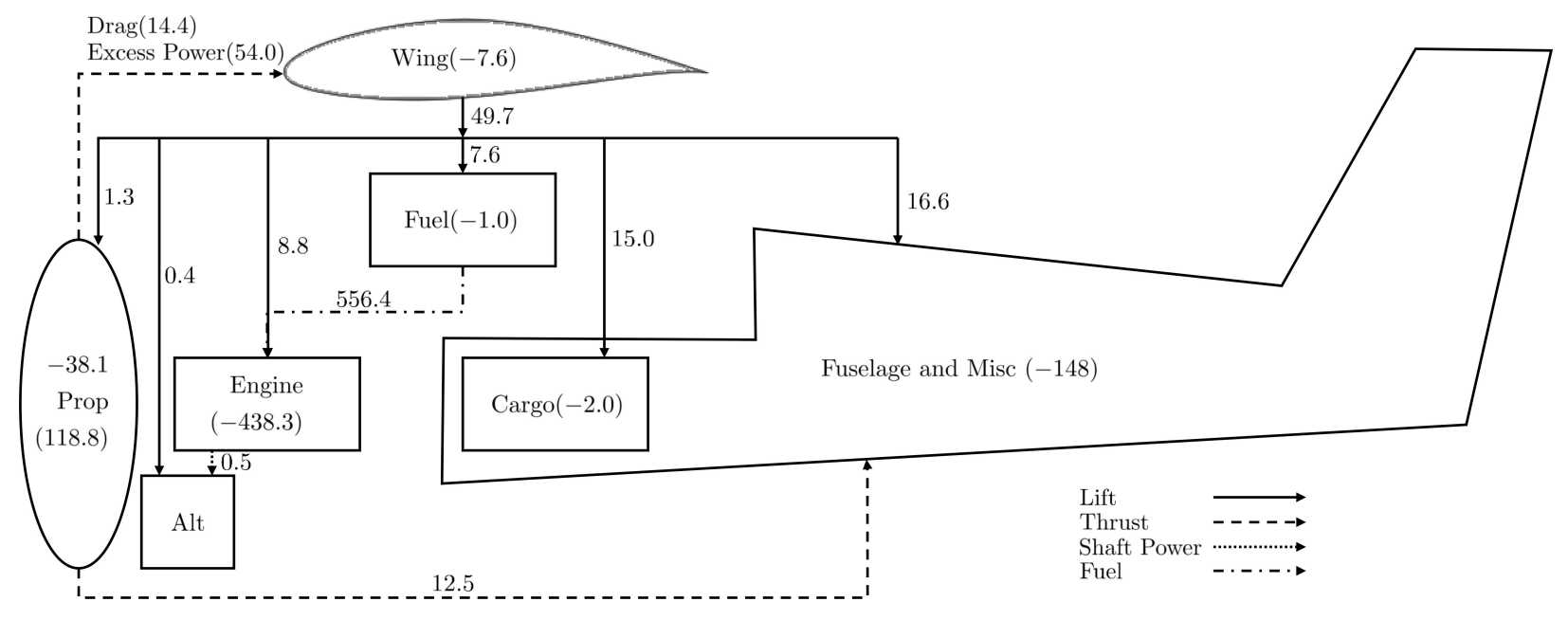

Figure 9: Exergy Flow Diagram of a light aircraft [76] [77]

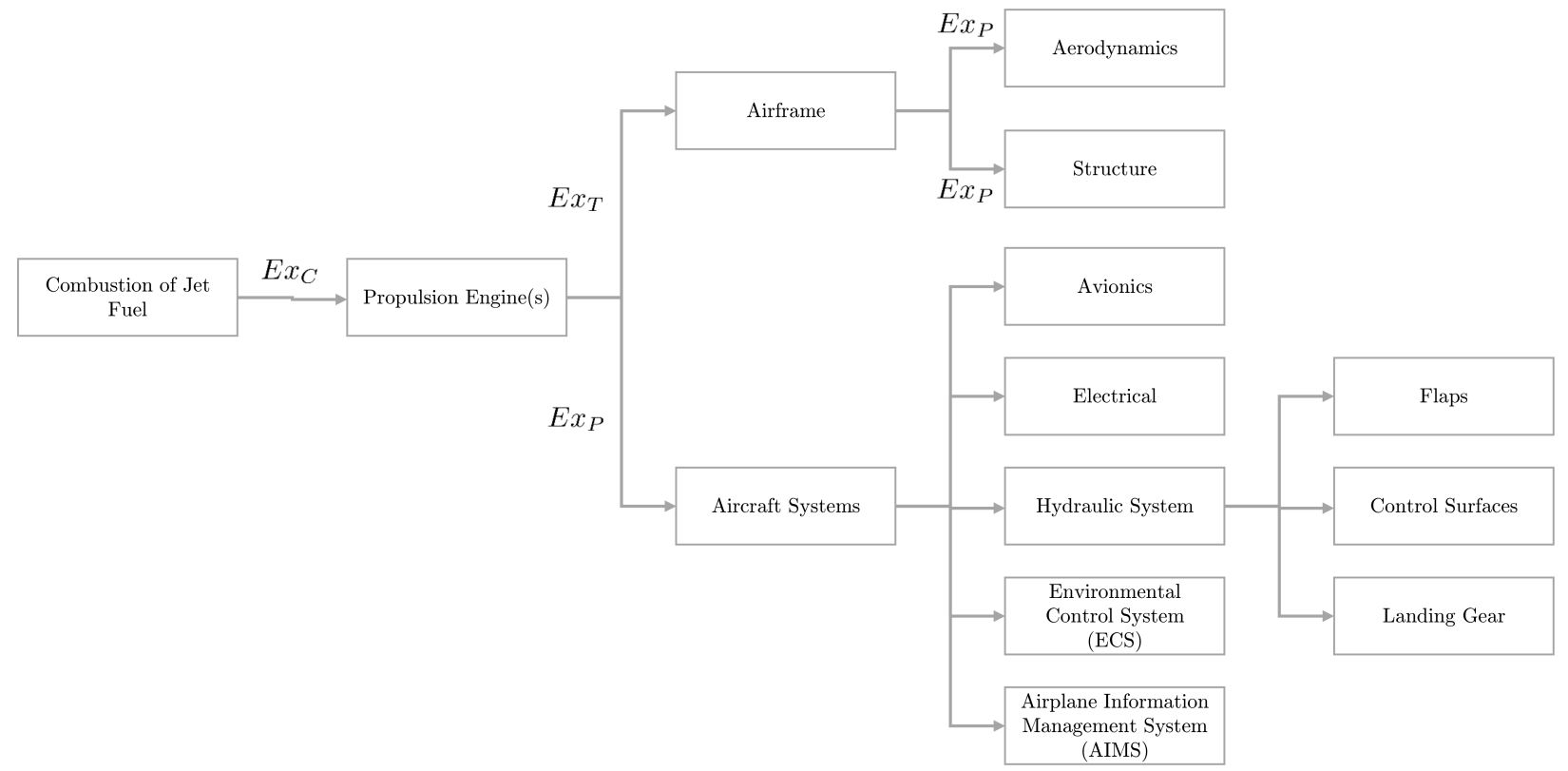

Figure 10: Exergy Flow through a generic commercial aircraft 


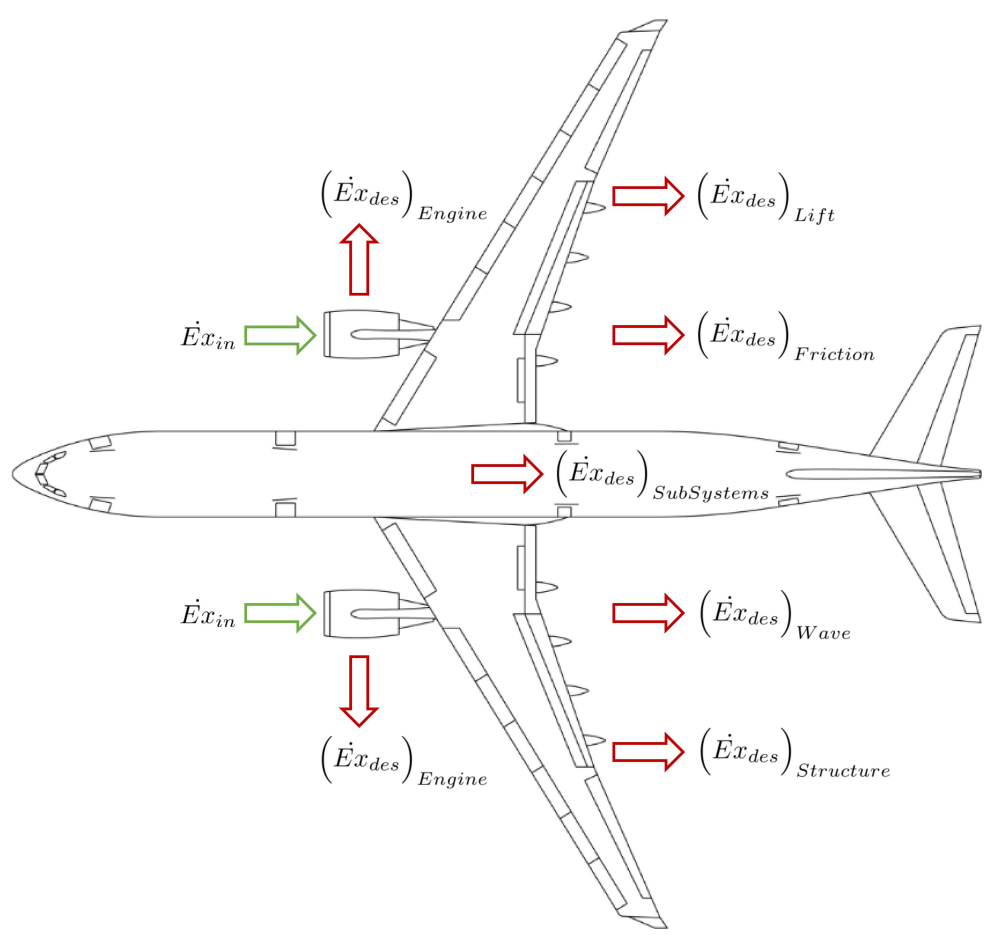

Figure 11: Exergy Flow Diagram of a generic commercial aircraft, derived from Camberos [29]

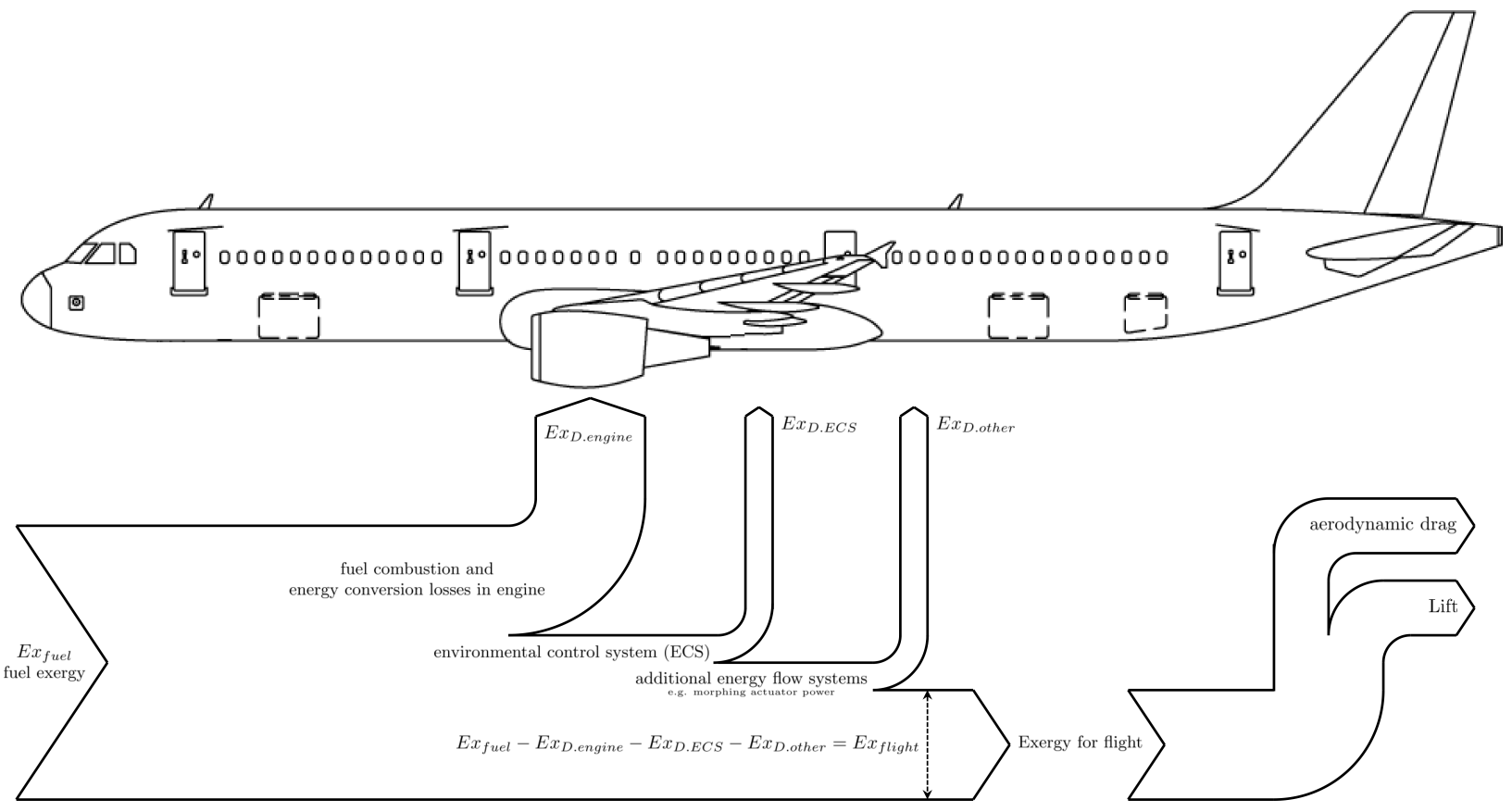

Figure 12: Grassmann diagram for the exergy flow through an aircraft, highlighting irreversibility locations 


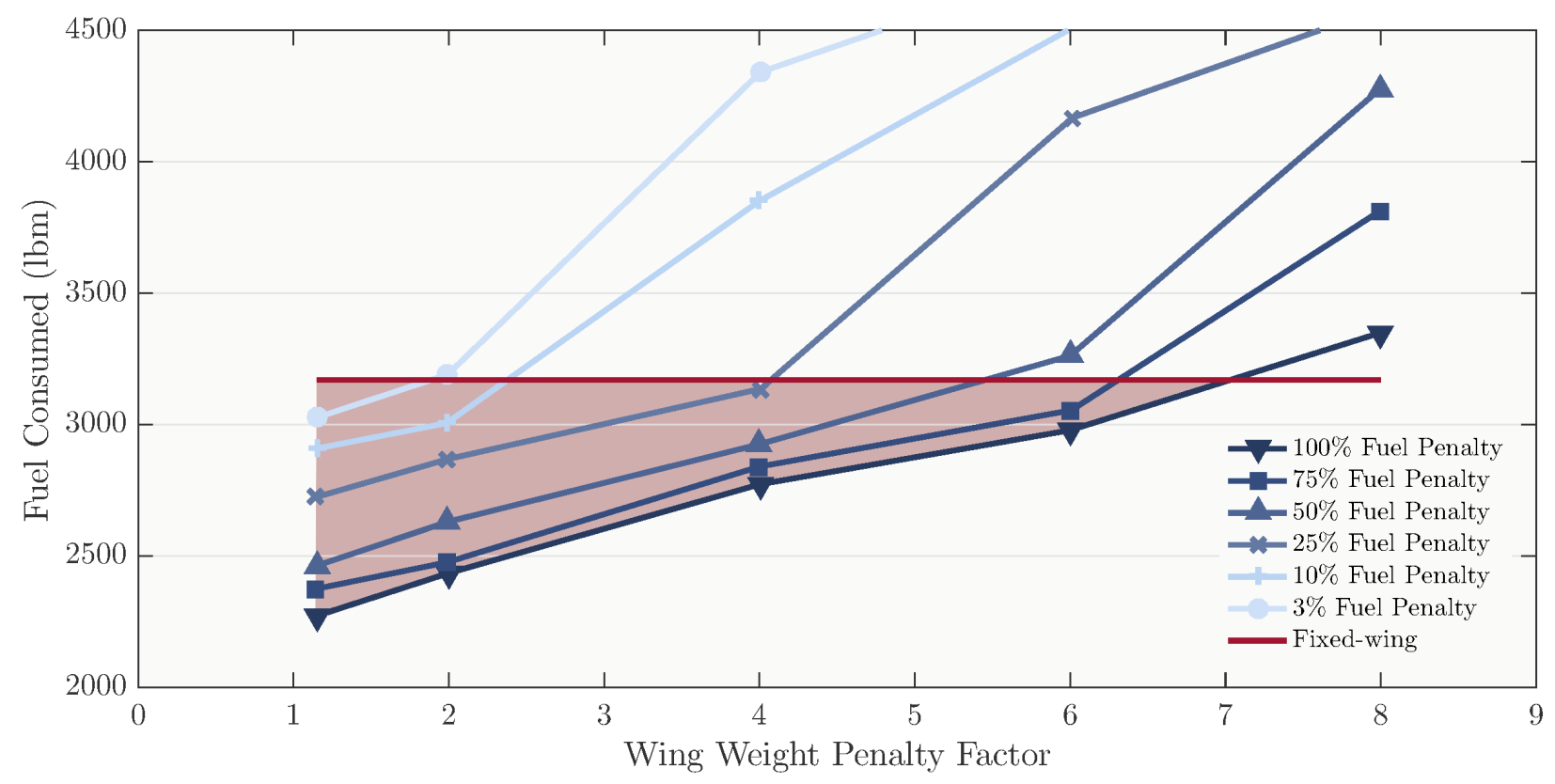

Figure 13: Sensitivity analysis of morphing wing effectiveness, Butt [28]

$$
\begin{aligned}
& \text { the ECS, fuel loop system, vapour compression loop system, electrical systems, central hydraulics systems, oil loop } \\
& \text { system and flight control systems. }
\end{aligned}
$$

\section{Improvement and Optimization Studies}

An example of multi-disciplinary integrated design with the use of an empirical exergy model, Doty [38] showed a surrogate model for a wing and turbo-fan engine, provided benefits from an exergy destruction point of view when compared to individually optimised sub-systems. Doty also compared the results of a first law analysis against that of a second law exergy analysis, commenting that the second law approach showed which optimisations were actually feasible (the concept of building directionality into the method).

The conclusions made by Doty [38] regarding the optimisation of integrated systems, echo those of Riggins [89] [90] who performed integrated system exergy analysis mainly on hypersonic vehicles. Riggins was also involved with the work of Marley [68] who took a lumped parameter model of a single-spool turbojet engine. The work highlighted under what conditions the steady exergy analysis methods can be applied to the transient operation of a turbojet engine. Marley [68] concluded that steady exergy analysis calculated engine thrust does track the actual thrust during transient manoeuvres, through two analysis on a full aircraft and engine in different control volumes as seen in Fig. 

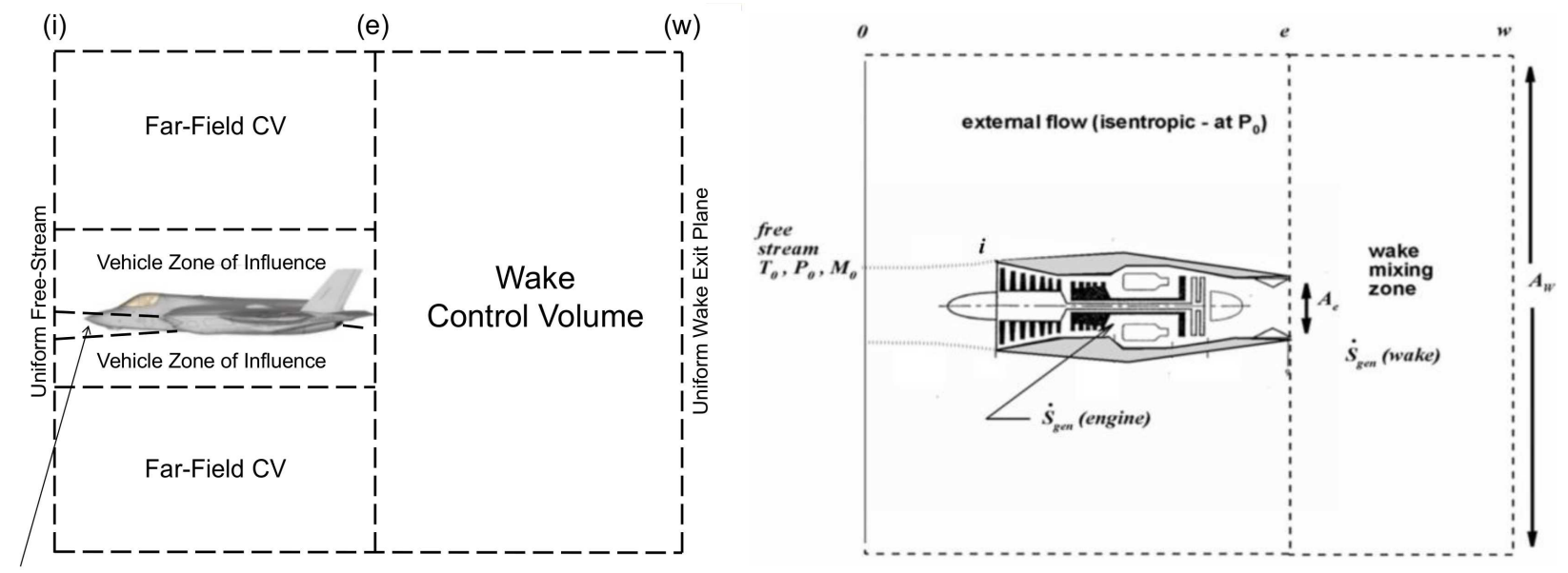

Propulsion Zone of Influence

Figure 14: Control volume definition for Marley study into entropy generation regions [68]

Riggins' [87] work on hypersonics is also documented in Camberos' textbook [31], where Riggins discusses how exergy analysis and optimisation provides significant advances in aerospace vehicle design, especially of hypersonic flight, where there is a demand for a thorough and systematic integration of all sub-systems. In this work, Riggins compared the output of two entropy based optimisation routines for the vehicle against a known set of design variables that yielded a maximum vehicle performance. The two entropy based method were (1) inclusive of the vehicle only availability and (2) included (1) but with the far field wake entropy generation. Riggins showed using a simple academic example (Fig. 15) that for hypersonic vehicles the wake entropy generation can be three to five times larger than entropy generation associated with the vehicle itself (see Fig. 16), thus the far-field volume must always be included in analysis.

\section{Mapping Exergy over the Variable Flight Envelope}

Ground based systems, such as power stations, which are typical subjects of exergy analysis have a relatively consistent external environment, so can be assumed to have a constant reference state. The majority of exergy analysis applied to aerospace vehicles makes the same assumption, whether this be a ground based propulsion system or assuming the complete vehicle operates only in the dominant cruise phase of flight. It is accepted that the maximum thrust obtainable from combustion is dependent on the composition of the environment in which the system operates, exergy analysis broadens this to any transfer of heat or work being dependent on the environment. In such a way, when exergy analysis is extended for use in aerospace applications it is evident that the external environment should be far from constant through the mission profile. The thermodynamic variables of temperature and pressure will significantly vary at sea level when compared to aircraft cruising altitude.

The reference state difficulty associated with aerospace exergy analysis has been studied by both Dincer [37] and Berg [22] [23] [24].

At a more fundamental level, Sciubba [97] states in relation to chemical exergy selecting a set of reference substances and determining their average concentration in the earths crust. These reference substances are the basis for the calculation of the exergy of the individual chemicals. The problem of how to identify a convenient "average composition" of the lito- hydro- and lower atmosphere was debated. Small differences in the reference elements produce substantial differences in the exergy values for most practical metals and fuels. At present, in practice all exergy calculations are based on the reference environment published by Szargut [103]

Gandolfi [50] mapped a complete flight mission of a commercial aircraft, identifying exergy destruction at different phases of flight. Fig. 17 are the results Gandolfi found for the distribution of irreversibilities among flight phases, where whilst cruise (assumed to be 40 minutes) is the largest destroyer of exergy, it does not overshadow the other phases as to make them negligible. A development of Gandolfi's work would be to study the actual flight missions of 


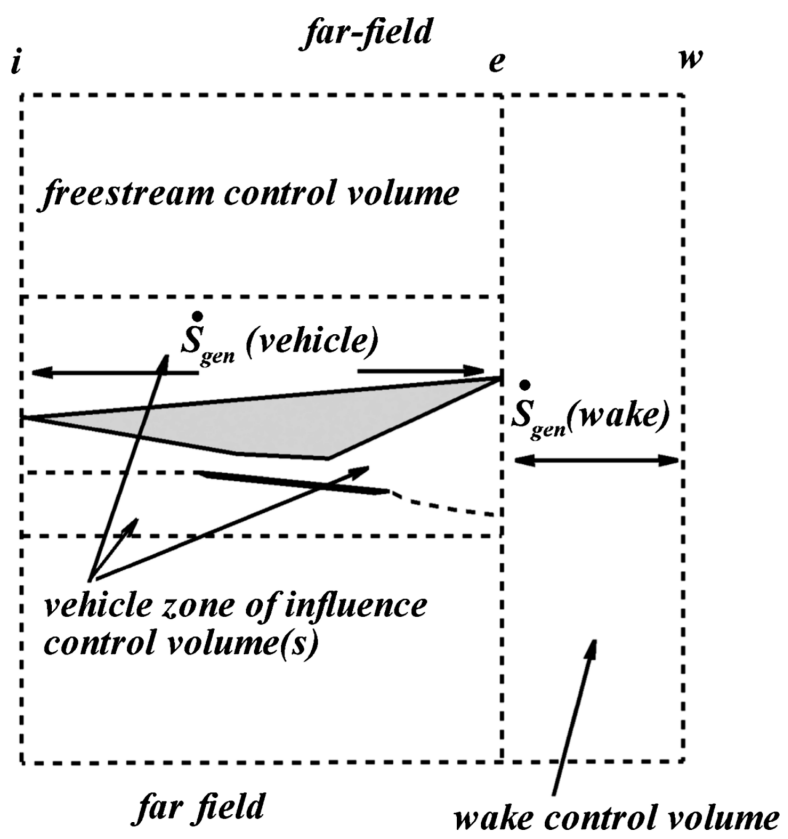

Figure 15: Global control volume showing constituent vehicle, freestream, and wake control volumes by Riggins [87]

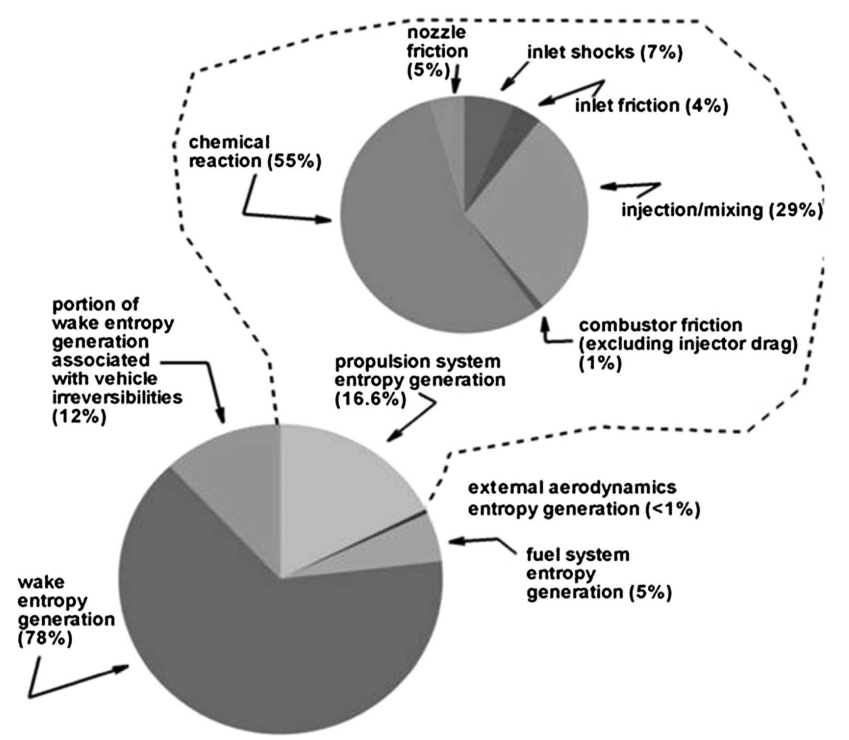

Figure 16: Detailed breakdown of instantaneous exergy losses at beginning of mission [87] 


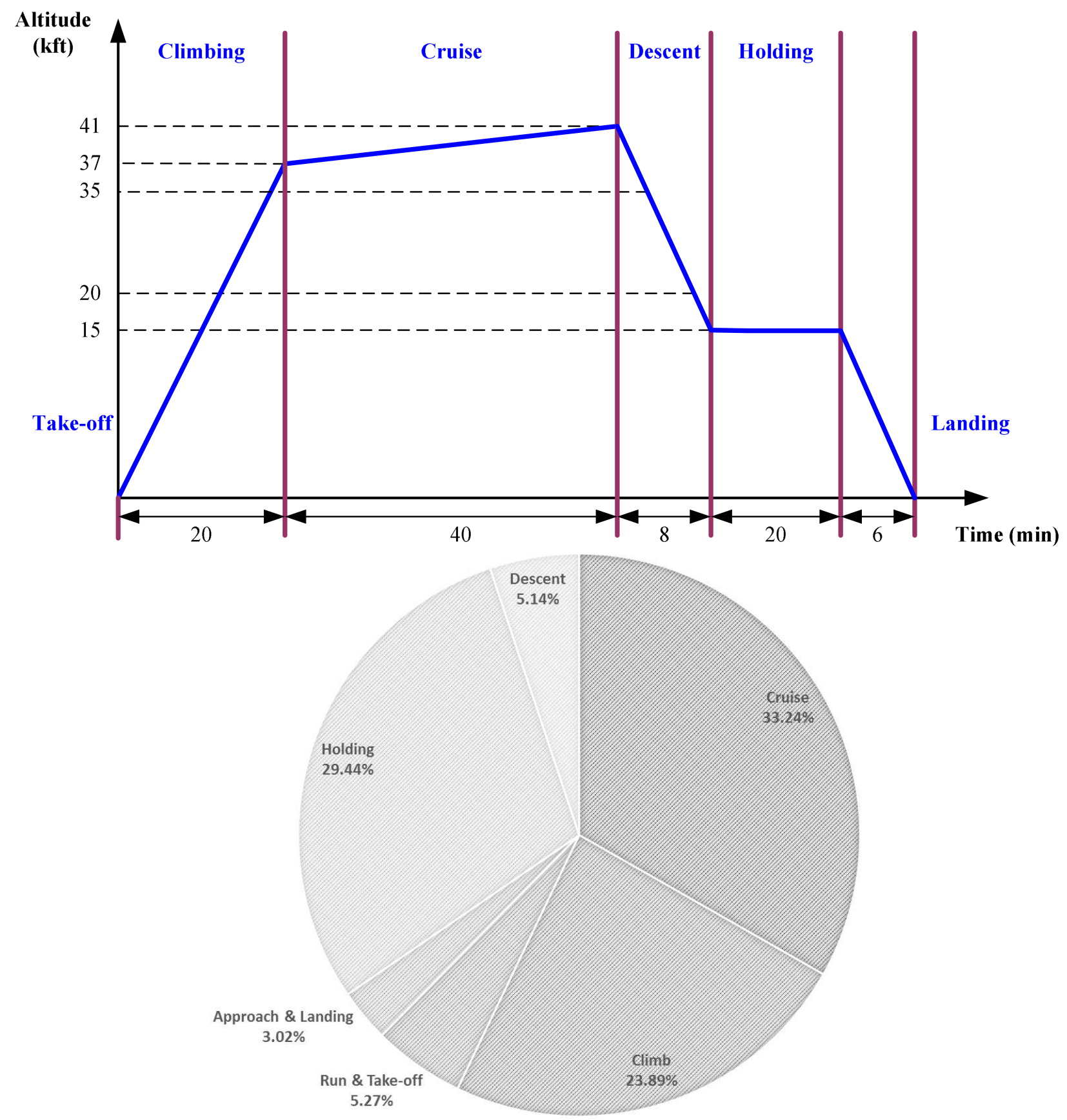

Figure 17: Distribution of losses as a percentage of exergy in different phases of flight as stated by Gandolfi [50] 


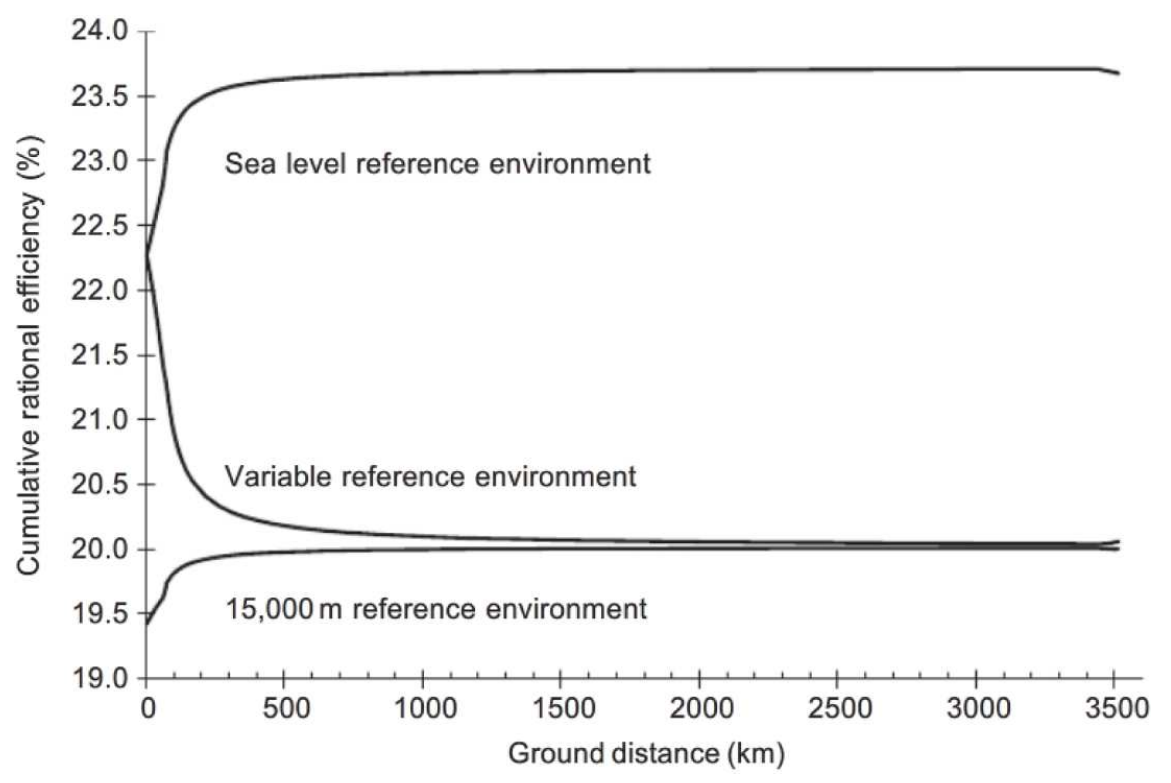

Figure 18: (a) Turbojet cumulative rational efficiency for various reference states [37]

airlines, as each aircraft can be used for a variety of different missions, often being used for missions the aircraft was not primarily designed for.

Dincer [37] adapted the work of Clarke [34] on theoretical analysis of aircraft turbojet power plants, to include a variable reference state, which Dincer compared against constant reference states at sea level and cruise altitude. The chosen comparison metric was cumulative rational efficiency (Equation 25) a function of the thrust power extracted, $P_{T}$ and the input exergy from fuel, $X_{i n}$. Dincer defined this as found that irregularities in the instantaneous efficiencies with flight distance are put into better perspective in terms of their impact on engine efficiency over an entire flight by weighting them by this ratio. Thus, short phases of flight such as take off, where the turbojet is running at a higher efficiency does not cloud the dominant phase of cruise flight.

$$
\psi_{\text {cum }}=\frac{\int_{0}^{t} P_{T}(t) d t}{\int_{0}^{t} X_{\text {in }}(t) d t}
$$

Fig. 18 displays the compared variable reference state and two constant states at sea level and cruise altitude. The sea level reference state can be seen to over estimate the efficiency of the turbojet when compared to the variable model, as well as having an inverse increase in efficiency during climb. This increase of efficiency during climb is an illusion of negative exergy in the incoming airflow, which occurs due to the growing discrepancy between the modelled sea level state and the actual state at altitude. The cruise altitude reference state creates a positive illusion of exergy during the climb phase, starting from a fictitiously low engine efficiency. However as the flight mission is dominated by cruise the plateau efficiency is close to that of the variable reference environment. Dincer [37] concludes that the variable reference state should be used for aerospace power unit applications, with a cruise altitude constant reference state only being used where the complexity of the variable reference state is not feasible.

Dincer modelled a flight mission dominated by the cruise phase of flight, which may be suitable for commercial flights. For a military flight mission there is typically no dominating flight phase as such the only option would be to use a variable reference state, or else errors in both numerical accuracy and predicted trends would be more evident with the constant state model.

Etele [44] conducted a similar analysis to Dincer on varying reference states $\left(T_{0}, P_{0}\right)$ by taking a turbojet engine and modelling the sensitivity of exergy efficiencies to the reference environment. Comparing analysis using reference states based on ground level, cruise altitude and one that varies the conditions based on flight phase. In contrast to the conclusions of Dincer, Etele was able to show that the exergy efficiency of a simplistic approach (ground level or 


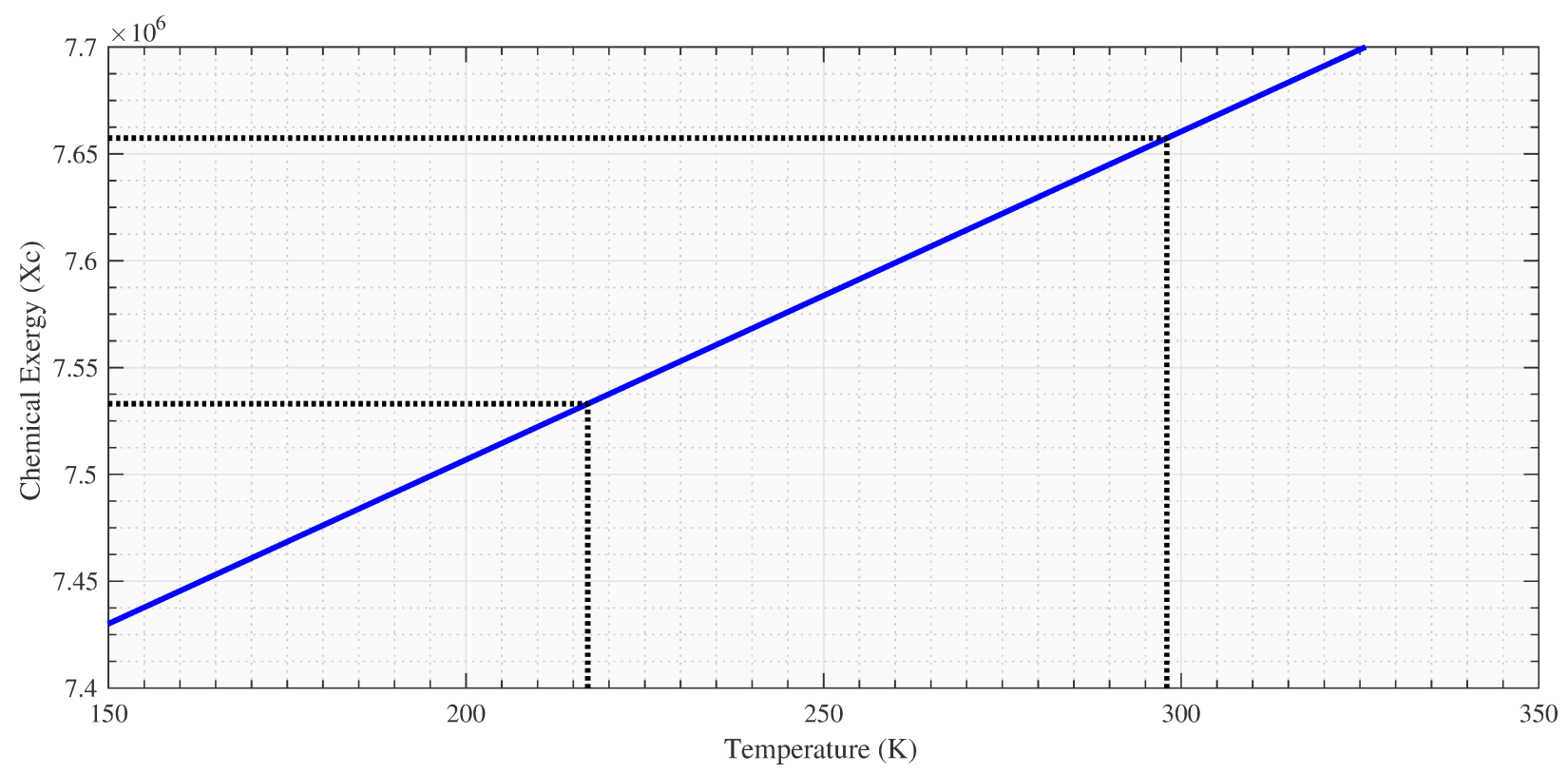

Figure 19: Variance of fuel chemical exergy with environment temperature [58]

cruise reference) gave a similar result to that of the complex variable reference state. However, the work of Berg [22] [24] on time-variant exergy analysis concluded that for a complete system mapping the vehicle exergy must allow for time variant analysis. Such an analysis allows for temporary storage of exergy. Berg undertakes a time-variant analysis in a similar method to that of Gandolfi [50] [51], where the phases of flight are broken down, and reference environment parameters are obtained for each of the broken down phases. Unlike Gandolfi, Berg is able to validate his results against a simple UAV model [22] and then through a more complex commercial aircraft mapping [24].

The initial exergy reserves are calculated by the exergy of the jet fuel and/or batteries on board the aircraft. The exergy of these sources is then mapped through each conversion process with the exergy destruction highlighted at each stage, to the point of complete exergy destruction.

Hayes [58] noted that assuming a constant enthalpy of formation and standard molar entropy with pressure variation, the chemical exergy of the fuel changes with altitude (variable temperature) as shown in Fig. 19, where the sea level chemical exergy content (at 298K) and cruise flight exergy content (at 217K) are highlighted. This relationship suggests a decrease in propulsion efficiency with increasing altitude, where in cruise the exergy available is $98 \%$ that available at sea level. A trend that is comparable to that seen with in use turbofan engine efficiencies.

The combustion of standard commercial aircraft fuel, Jet $\mathrm{A}\left(\mathrm{C}_{12} \mathrm{H}_{23}\right)$, is given as follows:

$$
\mathrm{C}_{12} \mathrm{H}_{23}+17.75 \mathrm{O}_{2} \rightarrow 12 \mathrm{CO}_{2}+11.5 \mathrm{H}_{2} \mathrm{O}
$$

Assuming environment of $T_{0}=298 \mathrm{~K}$ and $P_{0}=100 \mathrm{kPa}$ standard composition of air for $x_{k}$, the exergy released in combustion of Jet A can be obtained using equation 14

$$
E x_{c}=\mu_{C_{12} H_{23}, T M}+17.75 \mu_{O_{2}, 0}-12 \mu_{C_{2}, 0}-11.5 \mu_{H_{2} O, 0}=44.34 M J / k g
$$

\section{Developing Exergy for Future Aerospace Application}

Since the development of the de Havilland Comet (the first commercial jet liner) in 1949, the energy intensity ${ }^{10}$ for each aircraft evolution has reduced, see Fig. 20. The Advisory Council for Aviation Research and Innovation in

\footnotetext{
${ }^{10}$ Energy Intensity is a measure of aircraft fuel economy, stating the amount of energy required to move one passenger one kilometre
} 


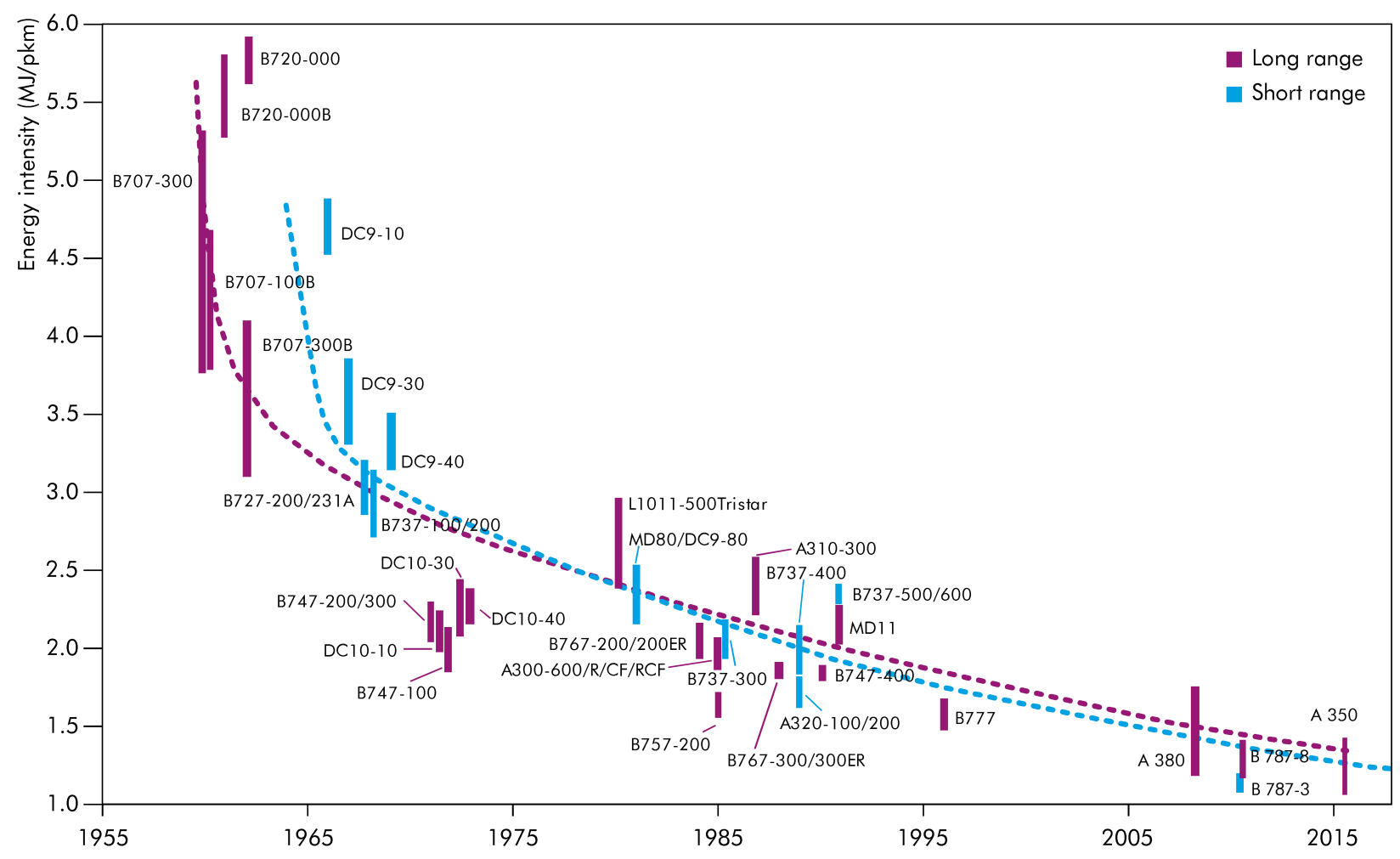

Figure 20: Evolution of aircraft energy intensity [59]

Europe (ACARE) [2] attributes this reduction to five major challenges for every future aircraft; (1) to meet market and societal needs, (2) to maintain and extend industrial leadership, (3) to protect the environment and the energy supply, (4) to ensure safety and security and (5) to prioritise research, test capabilities and education.

To protect the environment and the energy supply ${ }^{11}$, the European Commission has outlined an acceptable pace for emissions reduction in Flightpath 2050 [45]. In response, ACARE have laid out a set of targets ${ }^{12}$ that will enable the aims of Flightpath 2050 to be achieved. One of these targets states that relative to the capabilities in 2000, by 2050; "CO2 emissions per passenger kilometre [are to be] reduced by 75\%, NOx emissions by $90 \%$ and perceived noise by 65\%" [2]. However, air transport is currently experiencing the fastest growth of any mode of transport [62], and by 2035 it is estimated the required number of passenger aircraft will increase by 109\% from 2015 levels [4]. Thus, to meet the environmental targets original equipment manufacturers (OEMs) need to commit to keeping the trend in efficiency improvements that is evident in the latest generation of aircraft.

The market driven needs of airlines are also pushing for lower energy intensity aircraft, as a reduction either means lower more competitive air fares or a higher profit margin as a result of the lower fuel costs.

Recent improvements in propulsion efficiency are evident in the Airbus A320 NEO (New Engine Option) where the development of Geared Turbofan (GTF) engines offers a 15\% reduction in fuel burn [60]. The enhancement of the aerodynamic efficiency was the driver for the introduction of span extension technologies on the folding wingtips on the Boeing 777X. Advances in structural efficiency are noticeable in the proliferation of composite materials technology which has increased from roughly $15 \%$ at the end of the 20th century to the Boeing 787 and Airbus A350 XWB where approximately $50 \%$ of the structure weight is composite [60]. 


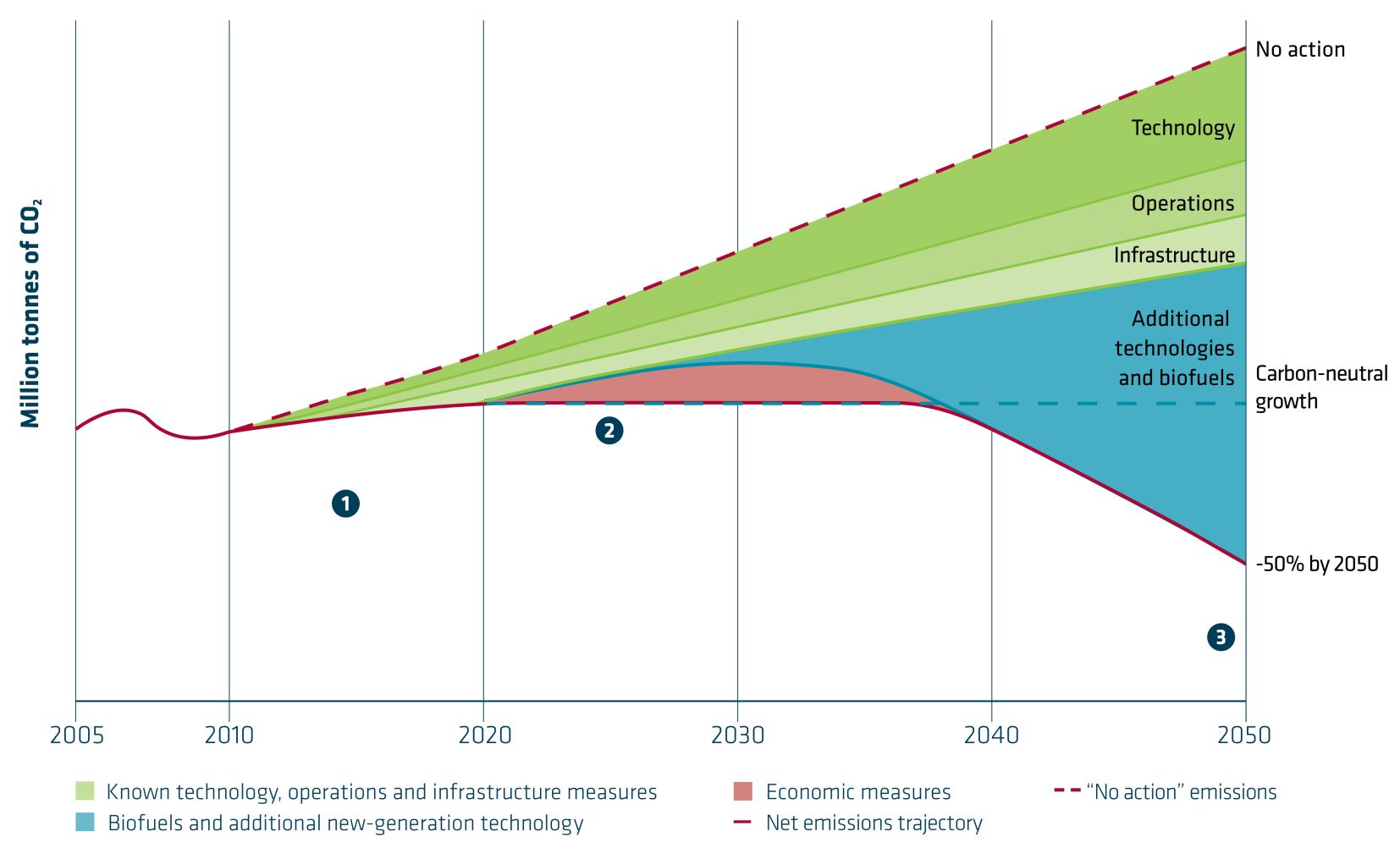

Figure 21: Predictions of measures to be taken to reduce carbon emissions [3]

\subsection{How to Meet the Requirements for Future Aircraft?}

These examples of evolutionary improvements to conventional configurations have contributed to aircraft now being 70\% more fuel efficient per seat kilometre than the de Havilland Comet [3]. A trend echoed by the European Environment Agency who note "the environmental performance of European transport is slowly improving, but there is still some way to go in decreasing oil consumption in transport" [46].

Thus the ACARE climate targets seem feasible, assuming technological developments continue at a similar rate. However, the International Energy Agency [59] has highlighted a trend that conventional aircraft configurations are near optimised, demonstrated by the plateauing improvements in energy intensity seen in Fig. 20.

Giurgiutiu [52] anticipates that research issues for future aircraft will be focused on "disruptive new and revolutionary structural concepts and unprecedented flight configurations". This premise is verified by ATAG [3] with the forecast that evolutionary improvements to current technology will account for less than $10 \%$ of the reduction in carbon emissions (see Fig. 21), with the majority of reductions expected from biofuels and additional new-generation technology.

Hence aircraft manufacturers are researching technologies and configurations that may provide the required performance improvements. Propulsion efficiency improvements are expected from next generation turbofan development as well as new concepts of open rotor engines and hybrid turbofan-electric propulsion as seen in the conceptual Airbus E-Thrust aircraft. Future aerodynamic efficiencies move away from the conventional swept aircraft configuration to High Aspect Ratio Wing (HARW)[25] and Prandtl box wing aircraft[32] to reduce vortex drag, blended wing body concepts to minimise aircraft surface area and thus parasitic drag and also laminar flow wing technology seen in the BLADE (Breakthrough Laminar Aircraft Demonstrator in Europe) project [60].

\footnotetext{
${ }^{11}$ To counter the contribution of aviation to global warming

${ }^{12}$ Replicated worldwide with targets from the Air Transport Action Group (ATAG) [3].
} 

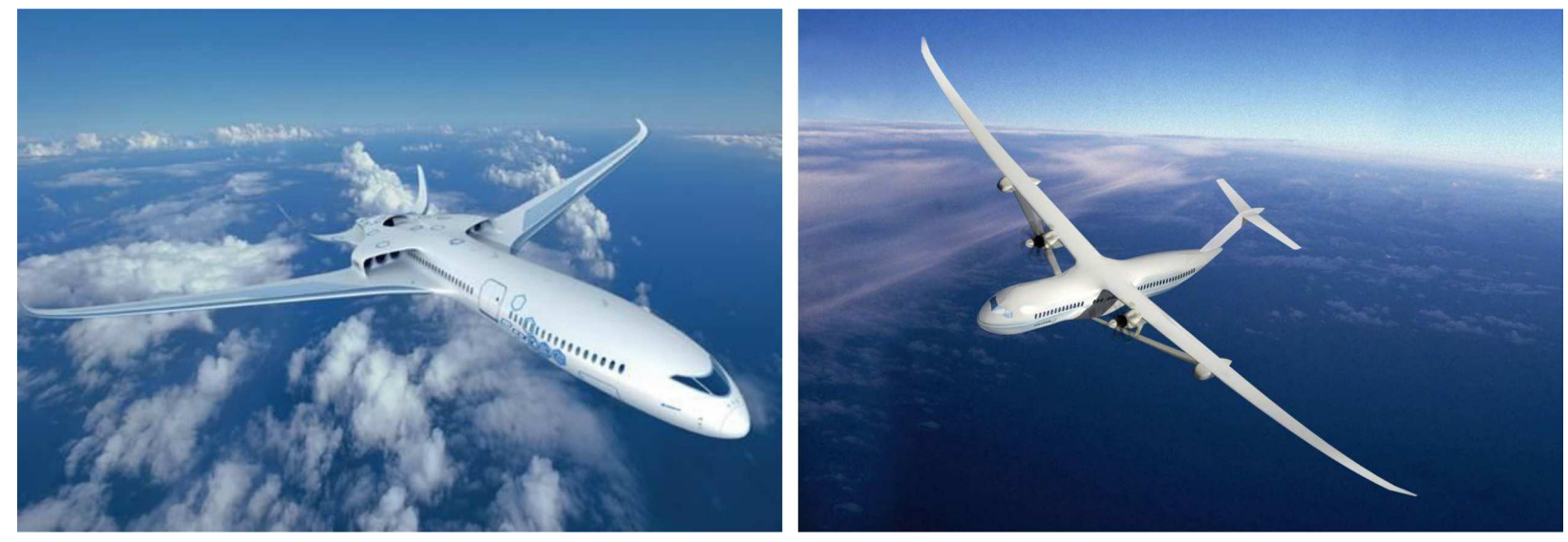

Figure 22: Airbus E-Thrust Concept (left) and NASA SUGAR Volt (right)

\subsection{Why an Energetics Based Approach is Applicable?} ous analytical process to supplement the lack of in-service performance information. This leads to the need for a methodology that can support design of the complete vehicle as a system of sub-systems in a common mathematical framework [30]. The Breguet method (equation 1) has proved adequate for current practice where sub-systems are designed independently of the top level system, thus the propulsion, aerodynamic and structural efficiencies can all e optimised separately. However, as commercial aircraft become more integrated vehicles, similar to what we see in military and hypersonic design today, performance optimisation needs to be considered at the top level, in order to account for the interactions between competing engineering disciplines, as failure to do so either places operating limits on systems or cost large sums of money to correct.

The Breguet equation does not provide a systematic way to trade performance metrics across disciplines. Hence, in order to facilitate highly integrated configurations, a new method of analysing the performance of an aircraft is required that allows inefficiencies in processes to be tracked across the whole system and the development of a decomposition strategy where all the components can be optimized to a system-level common metric. Such a holistic approach would treat all systems and interactions under one universal property that quantifies performance.

As an example, the performance benefit of a battery driven electric engine is difficult to assess against the conventional fuel driven turbofan. Not only do the two technologies require different system configurations with a battery storage requirement for electric propulsion (a mass that unlike jet fuel is not burnt off during flight), but the technologies have no common design metric.

\subsection{The Slow Adoption of Exergy Analysis}

Since the first use of the term available work by Gibbs [15], areas where exergy analysis has seen wide adoption are those processes dominated by thermodynamics, such as heat engines and power generation technology (internal combustion gas engines, steam power cycles, gas turbine cycles and renewable energy cycles), heat exchanges and heat networking, air conditioning systems, cryogenics and chemical processes. Direct references for these are not given as beyond the scope of this paper, but a comprehensive review of these technologies is provided by Sciubba [97] and Ayres [15].

Much of the work in the aerospace sector on exergy analysis is sub-system specific, focused in the propulsion community, given the traditional thermodynamic nature of the system, and as such read across from earlier uses of exergy analysis. However in the aerospace community, outside of propulsion, there has been a slow adoption of thermodynamic optimisation and the exergy method [97].

Ayres [15] gives the reason for the slow adoption of exergy being due to confusion and misunderstanding associated with thermodynamics, which essentially is generated due to many of the variables not being physical variables people can measure, including entropy, enthalpy, internal energy, heat, Gibbs free energy and exergy, whilst these are mathematically proven within the theory, they cannot be physically visualised. Noting the difficulty in explaining the 
concept of exergy, Zabihian [107] presented a paper purely focused on how to comprehend the concept of exergy and teach it to students, focusing on a more global understanding of the methodology rather than to just one application as many authors focus.

Edwards [42] argues the adoption of exergy methods has been slow in the field of combustion as exergies are approximately equal to the respective lower heating values, thus providing little benefit in real calculations.

A further obstacle is a consistent definition of exergy, which is exacerbated by the fact different authors have used various terms to refer to exergy and the term exergy for slight different purposes. Sciubba [97] and Ayres [15] provide examples of this including; available energy, Arbeitsfähigkeit (translated from German as working capacity), exergie (German), availability, available work, available useful work, useful energy, distinguishability and essergy (an abbreviation of essence of energy). Sciubba [97] states the accepted terminology is now exergy (with a few American authors still using the term availability). Justified by the definition of such work being based on energy meaning internal work, from the Greek en and ergon, and then changing the prefix to the Greek ex suggesting external application to work.

Working with an exergy metric would also require significant change to the design practice, as typically aircraft sub-systems are optimised for their individual requirements to the optimal operating conditions, irrespective to the top level optimisation and efficiency of the complete system. A critical part of implementing an exergy based approach is the development of a decomposition strategy where all the subsystem components can be optimized to a system-level common metric. This would be no easy task, as major sub-systems of aircraft are designed by different companies and incorperated at a higher level, such as the Airbus and engine suppliers relationship.

\subsection{Case Study - Next Generation Aircraft - High Aspect Ratio Morphing Wing}

To conclude the paper, below is a case study looking at how exergy analysis could be used for high aspect ratio morphing wing aircraft, aiming to trigger the readers mind with possible applications of such a method.

The majority of the Flightpath 2050[45] carbon reduction targets are assumed to occur due to additional technologies [3], a term which refers to technologies to be incorporated into aerospace systems that are not currently present and thus can't be improved from a base state. One such technology being considered is that of a morphing wing, where wing geometry is substantially changed in order to improve aircraft performance by either expanding the flight envelope or replacing conventional control devices for improved aircraft control. In effect it aims to allow real time vehicle adaptation, enabling optimal performance over this wider flight envelope. Morphing has been the subject of numerous studies over the past years, however an in-flight morphing device on an operational commercial aircraft is yet to be seen.

Morphing wing has been receiving more attention in recent years, due to the develop of next generation High Aspect Ratio Wing (HARW) commercial aircraft. As shown in Fig. 23 the trend is for higher aspect ratios for newer aircraft, primarily due to the increase in size and also the improvements of the lift the drag ratio.

The reason why morphing has not seen full-scale application in aerospace is not just due to the conservative design approach of the industry, but that changing the aerodynamic profile of a wing requires some form of actuation system, which must be powered and adds weight to the aircraft. Thus, any performance benefit morphing provides must outweigh the penalty due to additional weight and power. However, there is an absence of unifying metric that allows accurate comparison of the wide range of performance characteristics and different morphing technologies.

Also, consider a morphing device that significantly changes the aspect ratio of the wing. In current research the wing is treated as a rigid body [100], and the benefits of drag reduction are compared to the losses in morphing the wing to this position. However, the aeroelastic properties of the wing have also been significantly altered. Whilst affecting aircraft flight dynamics this also impacts structural dynamics including wing damping. Furthermore, any twist in the wing changes the lift distribution, all of which has to be considered under a single metric.

However, for a next generation HARW aircraft there are two major issues, firstly longer wings means a larger wing root bending moment under gust loading and secondly airports can only accommodate a limited wing span, a limit HARW aircraft will exceed. To counter the issues raised here, morphing wing may allow some form of Load Alleviation Devices (LAF) on the HARW to lower the wing root bending moment, and also allow wing folding at airports to accommodate gate restrictions, such as with the Boeing 777X. Designs of such devices again need to be considered under a single optimisation metric. It is therefore the authors opinion that exergy analysis and EGM may provide a capability that allows the realisation of this type of aircraft. 


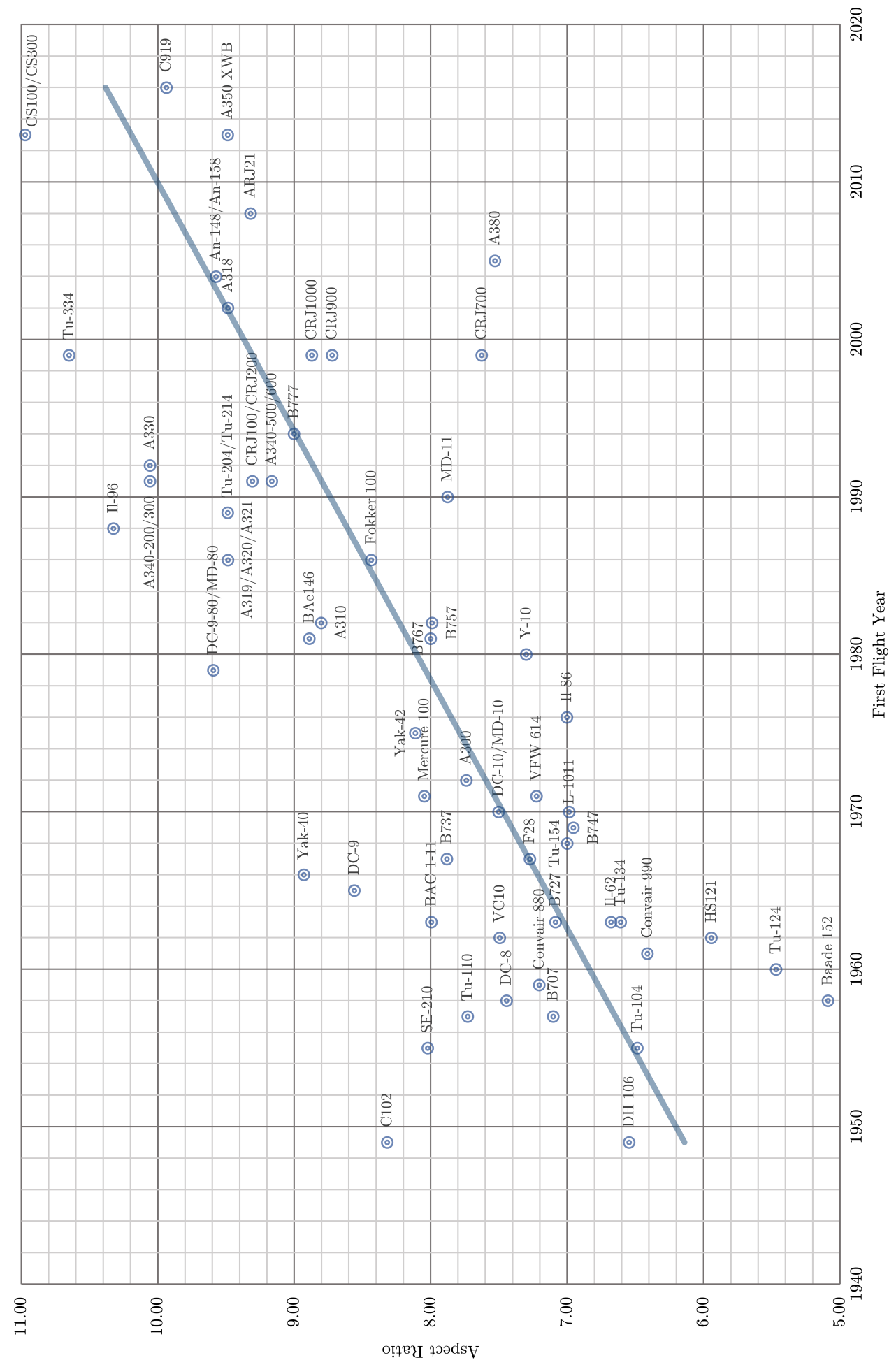

Figure 23: Aircraft Aspect Ratio against introduction year 


\subsection{Future Work in Exergy Based Aircraft Design}

The majority of exergy studies focus on steady-state performance analysis and adopt simplified models for flight

\section{Acknowledgements}

This research is supported by an Engineering and Physical Sciences Research Council (EPSRC) Industrial Cooperative Award in Science \& Technology (CASE) grant in collaboration with Airbus Group. dynamics and morphing, and estimate the exergetic cost of such devices in global performance terms such as weight and fuel burn penalties. As a result, the future direction for the development of exergy is the definition, calculation and analysis of dynamic exergy for flexible aircraft; where the effects of variables such as wing flexibility on stored strain energy and consequently exergy destruction will be explicitly studied. Developing a method for selecting the appropriate reference state will be another area of interest. The selection of a reference state is straight forward for steady-state analysis, however, in a dynamic scenario the exergetic content of the atmosphere over the course of a time-domain simulation must be considered to accurately account for a varying reference frame.

One of the common themes throughout the review is the fact that the work has been completed within the academic community on highly simplified examples. This is useful for proving the theoretical foundation of exergy analysis, but unless the method can be proven for more complex real world systems it is unlikely to be adopted into industry. umerous studies have been conducted into optimisation of specific aircraft sub-systems with exergy, such as the mental Control System (ECS), propulsion and wing / aerofoil geometry. The concept of complete aircraft mapping has been attempted by a few authors to show exergy destruction variation over different stages of aircraft flight.

\section{Conclusions}

This review has discussed the previous applications of exergy analysis in the aerospace sector, focusing primarily on commercial application. The limitations have been derived and discussed along with the potential benefits to further proliferation of this second law method in the aerospace community. It has been shown how exergy analysis and mapping exergy destruction provides:

- a consistent common currency to allow consistent accounting across sub systems

- loss-producing mechanisms can be readily mapped at the system level

- analysis space provides physically possible/meaningful bounds

- provides foundation for robust and efficient optimization

- should produce same result regardless of technique utilised, and also match the results of first law implicit methods, but providing additional insight on top of this

- an understanding of how one system influences and interacts with other non-discipline specific sub-systems

\section{References}

[1] Adeyinka, O. B., Naterer, G. F., 2004. Modeling of Entropy Production in Turbulent Flows. Journal of Fluids Engineering 126 (6), 893.

[2] Advisory Council for Aviation Research and innovation in Europe, 2012. Realising Europe's vision for aviation Strategic Research \& Innovation Agenda - Executive Summary. Tech. rep., ACARE.

[3] Air Transport Action Group, 2012. A sustainable flightpath towards reducing emissions. Tech. Rep. November, Air Transport Action Group, UNFCCC Climate Talks, Doha.

[4] Airbus, 2016. Global Market Forecast - Mapping Demand 2016-2035. URL http://www . airbus. com/company/market/forecast/

[5] Alabi, K., Ladeinde, F., 2007. Utilizing CFD-based exergy calculations in the design/optimization of a complete aircraft system. In: Collection of Technical Papers - 45th AIAA Aerospace Sciences Meeting. Vol. 20. pp. 13657-13671.

[6] Alabi, K., Ladeinde, F., Moorhouse, D. J., Camberos, J. A., 2006. Assessing CFD modeling of entropy generation for the air frame subsystem in an integrated aircraft design/synthesis procedure. In: Proceedings of the 44th AIAA Aerospace Sciences Meeting and Exhibit. No. January. pp. 1-19.

[7] Alabi, K., Ladeinde, F., Moorhouse, D. J., Camberos, J. A., Brook, S., 2006. A comparison of empirical and CFD based exergy modelling for the airframe. In: ICAS. pp. 1-10. 
for aircraft air frame subsystems. International Journal of Thermodynamics 9 (4), 193-205.

[9] Alabi, K., Ladeinde, F., Von Spakovsky, M. R., Moorhouse, D. J., Camberos, J. A., 2007. An advanced exergy and energy simulation tool for large-scale design/optimization of aerospace systems. In: Collection of Technical Papers - 39th AIAA Thermophysics Conference. Vol. 1. pp. $465-481$.

[10] Anderson, J. D., 1999. Aircraft performance and design, 1st Edition. WCB/McGraw-Hill.

[11] Arntz, A., Atinault, O., dec 2015. Exergy-Based Performance Assessment of a Blended WingBody with Boundary-Layer Ingestion. AIAA Journal 53 (12), 3766-3776.

[12] Arntz, A., Atinault, O., Destarac, D., Merlen, A., 2014. Exergy-based aircraft aeropropulsive performance assessment: CFD application to boundary layer ingestion. In: 32nd AIAA Applied Aerodynamics Conference. American Institute of Aeronautics and Astronautics Inc.

[13] Arntz, A., Atinault, O., Merlen, A., dec 2014. Exergy-Based Formulation for Aircraft Aeropropulsive Performance Assessment: Theoretical Development. AIAA Journal, 1-13.

[14] Arntz, A., Hue, D., 2015. Exergy-Based Performance Assessment of the NASA Common Research Model. AIAA Journal 54 (1), 1-13.

[15] Ayres, R. U., Ayres, L. W., Martinas, K., 1996. Eco-Thermodynamics: Exergy and Life Cycle Analysis, 49.

[16] Bauer, B., 1970. Critical remarks on the definition of jet engines propulsive efficiency (in German). Z. Flugwiss 18 (5), 158-171.

[17] Bejan, A., 1995. Entropy Generation Minimization: The Method of Thermodynamic Optimization of Finite-Size Systems and Finite-Time Processes, 1st Edition. CRC Press.

[18] Bejan, A., 1999. Role for exergy analysis and optimization in aircraft energy-system design. American Society of Mechanical Engineers, Advanced Energy Systems Division (Publication) AES 39, 209-217.

[19] Bejan, A., jun 2002. Fundamentals of exergy analysis, entropy generation minimization, and the generation of flow architecture. International Journal of Energy Research 26 (7), 545-565.

740 [20] Bejan, A., 2003. Constructal theory: Tree-shaped flows and energy systems for aircraft. Journal of Aircraft 40 (1), $43-48$.

[21] Bejan, A., dec 2012. Entropy Generation Minimization, Exergy Analysis, and the Constructal Law. Arabian Journal for Science and Engineering 38 (2), 329-340.

[22] Berg, F., 2013. Principles for Aircraft Exergy Mapping submitted by. Ph.D. thesis, University of Bath.

[23] Berg, F., Balchin, M., Keogh, P., oct 2011. Elucidation of Aircraft Energy Use Through Time-Variant Exergy Analysis. Tech. rep.

745 [24] Berg, F., Balchin, M., Keogh, P., jul 2013. New Principles for Dynamic Aircraft Exergy Mapping. Journal of Aircraft 50 (4), 1088-1098.

[25] Bradley, M. K., Allen, T. J., Droney, C. K., 2015. Subsonic Ultra Green Aircraft Research Phase II - Volume I - Truss Braced Wing Design Exploration. Tech. Rep. NASA/CR2015-218704, National Aeronautics and Space Administration, Virginia.

[26] Brewer, K. M., 2006. Exergy Methods for the Mission-Level Analysis and Optimization of Generic Hypersonic Vehicles. Ph.D. thesis, Virginia Polytechnic Institute and State University.

[27] Brilliant, H., 1995. Analysis of scramjet engines using exergy methods. In: 31st AIAA/ASME/SAE/ASEE Joint Propulsion Conference and Exhibit. AIAA, p. 17.

[28] Butt, J., 2005. A study of morphing wing effectiveness in fighter aircraft using exergy analysis and global optimization techniques. Ph.D. thesis, Virginia Polytechnic Institute and State University.

[29] Camberos, J. A., 2016. Analysis \& Design Using Exergy AFRL at a Glance. Tech. Rep. June.

755 [30] Camberos, J. A., Moorhouse, D. J., 2009. Systems engineering in terms of exergy. International Journal of Aerospace Engineering.

[31] Camberos, J. A., Moorhouse, D. J., 2011. Exergy Analysis and Design Optimization for Aerospace Vehicles and Systems, 1st Edition. American Institute of Aeronautics and Astronautics.

[32] Cavallaro, R., Demasi, L., 2016. Challenges, Ideas, and Innovations of Joined-Wing Configurations: A Concept from the Past, an Opportunity for the Future. Progress in Aerospace Sciences 87, 1-93.

760 [33] Cengel, Y. A., Boles, M. A., 2010. Thermodynamics: An Engineering Approach, seventh Edition. McGraw Hill Education.

[34] Clarke, J. M., Horlock, J. H., 1975. Availability and propulsion. ARCHIVE: Journal of Mechanical Engineering Science 1959-1982 (vols 1-23) 17 (4), 223-232.

[35] De Oliveira, S., 2013. Exergy method for conception and performance evaluation of aircraft systems. Ph.D. thesis, London.

[36] De Oliveira, S., 2013. Exergy: Production, Cost and Renewability. Green Energy and Technology. Springer London, London.

765 [37] Dincer, I., Rosen, M. A., 2013. Exergy Analysis of Aircraft Flight Systems. In: Exergy - Energy, Environment, and Sustainable Development, 2nd Edition. Elsevier, Ch. 19, pp. 383 - 392.

[38] Doty, J. H., Camberos, J. A., 2010. Statistical, modular systems integration using combined energy \& exergy concepts. In: 48th AIAA Aerospace Sciences Meeting Including the New Horizons Forum and Aerospace Exposition.

[39] Doty, J. H., Camberos, J. A., Moorhouse, D. J., 2008. Benefits of exergy-based analysis for aerospace engineering applications: Part 1. In: 40th AIAA Thermophysics Conference.

[40] Doty, J. H., Camberos, J. A., Moorhouse, D. J., 2009. Benefits of exergy-based analysis for aerospace engineering applications: Part 2. In: 47th AIAA Aerospace Sciences Meeting including the New Horizons Forum and Aerospace Exposition.

[41] Doty, J. H., Camberos, J. A., Moorhouse, D. J., 2009. Designed experiments: Statistical approach to energy- and exergy-based optimization. In: 39th AIAA Fluid Dynamics Conference.

775 [42] Edwards, C. F., 2012. Energy Tutorial: Exergy 101. Stanford University, GCEP Research Symposium, p. 26.

[43] Ehyaei, M., Anjiridezfuli, A., Rosen, M. A., 2013. Exergetic analysis of an aircraft turbojet engine with an afterburner. Thermal Science 17 (4), 1181-1194.

[44] Etele, J., Rosen, M. A., 2000. Exergy losses for aerospace engines - Effect of reference-environments on assessment accuracy. In: 38th Aerospace Sciences Meeting and Exhibit. AIAA, Reno, Nevada.

780 [45] European Commission, 2011. Flightpath 2050 Europes Vision for Aviation. Tech. rep., European Commission.

[46] European Environment Agency, 2014. Focusing on environmental pressures from long distance transport. Tech. Rep. 7, EEA Report No 7/2014.

[47] Figliola, R. S., 2005. Energy-Based Design Methodology for Air Vehicle Systems: Aerodynamic Correlation Study. Air Force Office of 
Scientific Research.

785 [48] Gaggioli, R. A., Paulus, D. M., 2002. Available EnergyPart II: Gibbs Extended. Journal of Energy Resources Technology 124 (2), 110.

[49] Gaggioli, R. A., Richardson, D. H., Bowman, A. J., 2002. Available EnergyPart I: Gibbs Revisited. Journal of Energy Resources Technology $124(2), 105$.

[50] Gandolfi, R., Pellegrini, L. F., Da Silva, G. A. L., De Oliveira, S., 2008. Exergy analysis applied to a complete flight mission of a commercial aircraft. In: 46th AIAA Aerospace Sciences Meeting and Exhibit.

[51] Gandolfi, R., Pellegrini, L. F., De Oliveira, S., 2010. More electric aircraft analysis using exergy as a design comparison tool. In: 48th AIAA Aerospace Sciences Meeting Including the New Horizons Forum and Aerospace Exposition.

[52] Giurgiutiu, V., 2009. Flight structures fundamental research trends and directions. Aeronautical Journal 113 (1144), $331-337$.

[53] Glansdorff, P., Jaumotte, A., Baland, J., 1955. Sur la puissance disponible et la rendement de propulsion des moteurs a reaction par jets (in French). Bull. Acad. Roy. Belg. Cl. des Sc. 5me Serie 41 (12), 1264-1280.

[54] Gouy, L. G., 1889. Sur l'énergie utilisable. Journal de Physique Théorique et Appliquée 8 (1), 501-518.

[55] Greene, G. C., sep 1989. An Entropy Method for Induced Drag Minimization. Tech. rep.

[56] Grönstedt, T., Irannezhad, M., Lei, X., Thulin, O., Lundbladh, A., nov 2013. First and Second Law Analysis of Future Aircraft Engines. Journal of Engineering for Gas Turbines and Power 136 (3), 031202.

[57] Hayes, D., Lone, M. M., Whidborne, J. F., 2016. Entropy Generation Minimisation and Exergy analysis approaches for aerospace applications - A review. In: 54th AIAA Aerospace Sciences Meeting, AIAA SciTech Forum. No. AIAA 2016-0866. San Diego, California, USA, pp. $1-18$.

[58] Hayes, D., Lone, M. M., Whidborne, J. F., Coetzee, E., jan 2017. Evaluating the Rationale for Folding Wing Tips Comparing the Exergy and Breguet Approaches. In: 55th AIAA Aerospace Sciences Meeting, AIAA SciTech Forum. American Institute of Aeronautics and Astronautics, Dallas, Texas. URL http://arc.aiaa.org/doi/pdf/10.2514/6.2017-0464

[59] International Energy Agency, 2009. Transport, Energy and CO2: Moving toward Sustainability. Tech. rep., International Energy Agency.

[60] Jupp, J. A., jan 2016. The design of future passenger aircraft â" the environmental and fuel price challenges 120 (1223), 37-60.

[61] Lee, J., Lukachko, S. P., Waitz, I. A., 2004. Aircraft and energy use. Encyclopedia of Energy 7, 29.

[62] Lee, J. J., Lukachko, S. P., Waitz, I. A., Schafer, A., nov 2001. Historical and future trends in aircraft performance, cost, and emissions. Annual Review of Energy and the Environment 26 (1), 167-200.

[63] Lewis, J., 1976. Propulsive efficiency from an energy utilization standpoint. Journal of Aircraft 13, $299-302$.

[64] Li, R., Qiu, X., 1992. Exergy Analysis of Aeroengine and its significance./Journal of Aerospace Power. Journal of Aerospace Power 7 (2), 169-172.

[65] Maltry, W., 1971. Beitrag zur Anwendung des Zweiten Hauptsatzes auf lufttechnische Prozesse (In German). Luft- u. Kältetechn 7, $227-230$.

[66] Markell, K. C., 2005. Exergy Methods for the Generic Analysis and Optimization of Hypersonic Vehicle Concepts. Ph.D. thesis, Virginia Polytechnic Institute and State University.

[67] Markell, K. C., Brewer, K. M., von Spakovsky, M. R., 2007. Exergy Methods Applied to the Integrated Mission-Level Analysis and Optimization of Hypersonic Vehicle Concepts. In: Volume 6: Energy Systems: Analysis, Thermodynamics and Sustainability. Vol. 6. ASME, pp. 265-277.

[68] Marley, C. D., Riggins, D. W., jan 2012. Exergy Analysis of a Turbojet Engine Modeled as a Lumped Parameter System. In: 50th AIAA Aerospace Sciences Meeting including the New Horizons Forum and Aerospace Exposition. American Institute of Aeronautics and Astronautics, Reston, Virigina.

[69] McGuire, S., 2016. Exergy Analysis of the NASA Common Research Model. Ph.D. thesis, Cranfield University.

[70] Memon, M. O., Wabick, K., Altman, A., Buffo, R. M., jul 2015. Wing Tip Vortices from an Exergy-Based Perspective. Journal of Aircraft $52(4), 1267-1276$.

[71] Moorhouse, D. J., Pratt, D. M., 2011. Methods to Optimize for Energy Efficiency Developed by Presented by : Wright- Patterson AFB .... Tech. Rep. May.

[72] Moorhouse, D. J., Sanders, B., Von Spakovsky, M. R., Butt, J., 2006. Benefits and design challenges of adaptive structures for morphing aircraft. Aeronautical Journal 110 (1105), 157-162.

830 [73] Naterer, G. F., Camberos, J. A., 2008. Entropy Based Design and Analysis of Fluids Engineering Systems, 1st Edition. CRC Press.

[74] Nixon, D., 2009. Fundamental Aerodynamics A Different Views. David Nixon.

[75] Oswatitsch, K., 1980. Contributions to the development of gasdynamics. Friedr. Vieweg \& Sohn Verlagsges GmbH.

[76] Paulus, D. M., Gaggioli, R. A., 2003. Rational objective functions for vehicles. Journal of Aircraft 40 (1), $27-34$.

[77] Paulus, D. M., Gaggioli, R. A., 2003. The Exergy of Lift and Aircraft Exergy Flow Diagrams. International Journal of Thermodynamics $6(4), 149-156$.

[78] Pellegrini, L. F., Gandolfi, R., Da Silva, G. A. L., De Oliveira, S., 2007. Exergy analysis as a tool for decision making in aircraft systems design. In: Collection of Technical Papers - 45th AIAA Aerospace Sciences Meeting. Vol. 23. pp. 16485-16496.

[79] Periannan, V., 2005. Investigation of the Effects of Various Energy and Exergy-Based Objectives / Figures of Merit on the Optimal Design of High Performance Aircraft System. Ph.D. thesis.

[80] Periannan, V., von Spakovsky, M. R., Moorhouse, D. J., 2006. Investigation of the Effects of Various Energy and Exergy-Based Figures of Merit on the Optimal Design of a High Performance Aircraft System. In: Advanced Energy Systems. Vol. 2006. ASME, pp. 337-347.

[81] Periannan, V., Von Spakovsky, M. R., Moorhouse, D. J., 2008. A study of various energy- And exergy-based optimisation metrics for the design of high performance aircraft systems. Aeronautical Journal 112 (1134), 449-458.

[82] Popov, A. V., Grigorie, L. T., Botez, R. M., Mamou, M., Mébarki, Y., jul 2010. Real Time Morphing Wing Optimization Validation Using Wind-Tunnel Tests. Journal of Aircraft 47 (4), 1346-1355.

[83] Rancruel, D. F., 2003. A Decomposition Strategy Based on Thermoeconomic Isolation Applied to the Optimal Synthesis / Design and Operation of an Advanced Fighter Aircraft System A Decomposition Strategy Based on Thermoeconomic Operation of an Advanced Fighter Aircraft System. Ph.D. thesis. 
[84] Rancruel, D. F., Von Spakovsky, M. R., 2004. Advanced fighter aircraft sub-systems optimal synthesis/design and operation: Airframe integration using a thermoeconomic approach. In: Collection of Technical Papers - 10th AIAA/ISSMO Multidisciplinary Analysis and Optimization Conference. Vol. 6. pp. 3403-3415.

[85] Riggins, D. W., 1997. Evaluation of performance loss methods for high-speed engines and engine components.

[86] Riggins, D. W., 1997. Thrust losses in hypersonic engines part 2: Applications. Journal of Propulsion and Power 13 (2), $288-295$.

[87] Riggins, D. W., Camberos, J. A., Wolff, M., Bowcutt, K., may 2013. Mission-Integrated Exergy Analysis for Hypersonic Vehicles: Methodology and Application. Journal of Propulsion and Power 29 (3), 610-620.

[88] Riggins, D. W., McClinton, C. R., Vitt, P. H., 1997. Thrust losses in hypersonic engines part 1: Methodology. Journal of Propulsion and Power 13 (2), 281-287.

[89] Riggins, D. W., Taylor, T. M., Moorhouse, D. J., 2006. The second-law based analysis of aerospace vehicle performance. In: Collection of Technical Papers - 44th AIAA Aerospace Sciences Meeting. Vol. 6. pp. 4020-4040.

[90] Riggins, D. W., Taylor, T. M., Terhune, L., Moorhouse, D. J., 2007. Methods for the design of energy efficient high speed aerospace vehicles. Aeronautical Journal 111 (1119), 297-309.

[91] Roth, B. A., 2000. A Theoretical Treatment of Technical Risk in Modern Propulsion System Design A Theoretical Treatment of Technical Risk in Modern Propulsion System Design. Ph.D. thesis, Georgia Institute of Technology.

[92] Roth, B. A., 2002. Work potential perspective of engine component performance. Journal of Propulsion and Power 18 (6), $1183-1190$

[93] Roth, B. A., 2004. Work transfer analysis of turbojet and turbofan engines. Proceedings of the 40th Joint Propulsion Conference, ....

[94] Roth, B. A., Mavris, D. N., jan 2003. Generalized model for vehicle thermodynamic loss management. Journal of Aircraft 40 (1), $62-69$. URL http: //arc.aiaa.org/doi/10.2514/2.3058

[95] Roth, B. A., Mavris, D. N., 2003. Method for propulsion technology impact evaluation via thermodynamic work potential. Journal of Aircraft 40 (1), 56-61.

[96] Schmitz, O., Hornung, M., sep 2013. Unified Applicable Propulsion System Performance Metrics. Journal of Engineering for Gas Turbines and Power 135 (11), 111201.

[97] Sciubba, E., Wall, G., 2007. A brief commented history of exergy from the beginnings to 2004. International Journal of Thermodynamics $10(1), 1-26$

[98] Simpson, A. P., Edwards, C. F., mar 2011. An exergy-based framework for evaluating environmental impact. Energy 36 (3), $1442-1459$.

[99] Smith, K., 2009. Morphing Wing Fighter Aircraft Synthesis / Design Optimization. Ph.D. thesis, Virginia Polytechnic Institute and State University.

[100] Smith, K., Butt, J., Von Spakovsky, M. R., Moorhouse, D. J., 2007. A study of the benefits of using morphing wing technology in fighter aircraft systems. In: Collection of Technical Papers - 39th AIAA Thermophysics Conference. Vol. 2. pp. $1497-1508$.

[101] Sohret, Y., Ekici, S., Altuntas, Ö., Hepbasli, A., Karakoç, T. H., 2016. Exergy as a useful tool for the performance assessment of aircraft gas turbine engines: A key review. Progress in Aerospace Sciences 83, 57-69.

[102] Stodola, A., 1945. Steam and Gas Turbines, 6th Edition. Peter Smith, New York.

[103] Szargut, J., Morris, D., Steward, F., 1988. Exergy analysis of thermal, chemical, and metallurgical processes, 1st Edition. Hemisphere Publishing Corporation.

[104] Tang, J., Bao, W., Yu, D., 2008. The Theoretical Treatment of Comparison of Performance between two scramjet engines with Energybypass based on Exergy Method. In: 46th AIAA Aerospace Sciences Meeting and Exhibit.

[105] Turan, O., 2014. Some Exergetic Measures of a JT8D Turbofan Engine. Journal of Automation and Control Engineering 2 (2), 110-114

[106] Von Spakovsky, M. R., 2003. The Use of Exergy and Decomposition Techniques in the Development of Generic Analysis and Optimization Methodologies Applicable to the Synthesis/Design of Aircraft / Aerospace Systems. Tech. rep., Virginia Tech.

[107] Zabihian, F., 2015. Educating Undergraduate Mechanical Engineering Students about Exergy Analysis. In: 122nd ASEE Annual Conference \& Exposition. American Society for Engineering Education, Seattle, Washington, pp. 26.570 .1 - 26.570.12. 
2017-08-05

\title{
Adopting exergy analysis for use in aerospace
}

\author{
Hayes, David
}

Elsevier

David Hayes, Mudassir Lone, James F. Whidborne, José Camberos, Etienne Coetzee, Adopting exergy analysis for use in aerospace, Progress in Aerospace Sciences, Volume 93, 2017, Pages 73-94

https://doi.org/10.1016/j.paerosci.2017.07.004

Downloaded from Cranfield Library Services E-Repository 